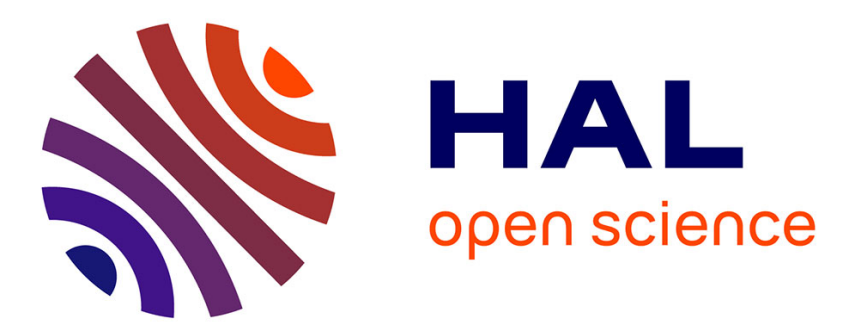

\title{
Efficient and robust computation of PDF features from diffusion MR signal
}

Haz-Edine Assemlal, David Tschumperlé, Luc Brun

\section{To cite this version:}

Haz-Edine Assemlal, David Tschumperlé, Luc Brun. Efficient and robust computation of PDF features from diffusion MR signal. Medical Image Analysis, 2009, pp.1-16. 10.1016/j.media.2009.06.004 . hal00410615

\section{HAL Id: hal-00410615 \\ https://hal.science/hal-00410615}

Submitted on 21 Aug 2009

HAL is a multi-disciplinary open access archive for the deposit and dissemination of scientific research documents, whether they are published or not. The documents may come from teaching and research institutions in France or abroad, or from public or private research centers.
L'archive ouverte pluridisciplinaire HAL, est destinée au dépôt et à la diffusion de documents scientifiques de niveau recherche, publiés ou non, émanant des établissements d'enseignement et de recherche français ou étrangers, des laboratoires publics ou privés. 


\title{
Efficient and Robust Computation of PDF Features from Diffusion MR Signal
}

\author{
Haz-Edine Assemlal, David Tschumperlé, Luc Brun \\ GREYC (CNRS UMR 6072), 6 Bd Maréchal Juin, 14050 Caen Cedex, France
}

\begin{abstract}
We present a method for the estimation of various features of the tissue micro-architecture using the diffusion magnetic resonance imaging. The considered features are designed from the displacement probability density function (PDF). The estimation is based on two steps: first the approximation of the signal by a series expansion made of Gaussian-Laguerre and Spherical Harmonics functions; followed by a projection on a finite dimensional space. Besides, we propose to tackle the problem of the robustness to Rician noise corrupting in-vivo acquisitions. Our feature estimation is expressed as a variational minimization process leading to a variational framework which is robust to noise. This approach is very flexible regarding the number of samples and enables the computation of a large set of various features of the local tissues structure. We demonstrate the effectiveness of the method with results on both synthetic phantom and real MR datasets acquired in a clinical time-frame.
\end{abstract}

Key words: Diffusion MRI, Gaussian-Laguerre, Spherical Harmonics, Rician noise, Variational framework.

\section{Introduction}

Diffusion Magnetic Resonance Imaging (dMRI) is an in-vivo method which captures images of water molecule diffusion. Its interesting ability to visualize human tissues with a high contrast and its extreme sensitivity to diagnose early stages of ischemic events and brain pathologies has been very useful in clinical applications and brain research so far [1, 2, 3]. Indeed, under the assumption that water molecules motion are restricted by the nerve fiver structures, the computation of the diffusion indexes leads to images which indirectly characterize the local micro-structure of fibers in each voxel. The MR signal acquisition is based on the pulsed field gradient spin-echo experiment (PFGSE) introduced by Stejskal and Tanner [4]. Until recently, most dMRI studies used the Stejskal and Tanner equation to analyse the signal attenuation [4],

$$
E_{g}=\frac{S_{g}}{S_{0}}=\exp \left(-\gamma^{2} g^{2} \delta^{2}(\Delta-\delta / 3) D\right)=\exp (-b D)
$$

This equation relates the normalized signal decay $E_{g}$ with the duration, time separation and strength of the magnetic field pulse gradients ( $\delta, \Delta$ and $g$ respectively), $\gamma$ the magnetogyric ratio, and the apparent diffusion coefficient (ADC) $D$. The symbols $S_{g}$ and $S_{0}$ respectively denote the diffusion signal decay at gradient $g$ and the baseline image without any gradient. LeBihan et al. [1] introduced Diffusion Weighted Imaging (DWI) which measures a scalar ADC along a single gradient direction $g$ from the signal decay. However, such an approach assumes that the diffusion is isotropic everywhere. Therefore, this model does not fit reliably the underlying micro-structure of tissues. Later, Basser et al. [5] introduced the Diffusion Tensor Imaging (DTI) which requires several acquisitions along at least 7

Email address: assemlal@greyc.ensicaen.fr (Haz-Edine Assemlal) directions of $\mathbf{g}$ to estimate the ADC as a symmetric, positivedefinite, 2nd-order tensor $D(\mathbf{g})$. DTI is then able to compute an angular measure of the diffusion. Despite its wide use, it assumes a displacement probability characterized by an oriented Gaussian Probability Diffusion Function (PDF). Consequently, DTI can only map a single orientation inside a voxel and fails in voxels having orientational heterogeneity [6]. Although high order methods have been introduced to better fit the ADC profile with more samples, one important issue remains: the ADC peaks do not necessarily yield the underlying main fiber orientations [7]. Besides, Eq.(1) describes a single population that exhibits unrestricted diffusion and can only capture a monoexponential decay which is therefore what most studies above assumed.

q-space: Built on top of the Stejskal-Tanner pulsed-field gradient spin-echo experiments [4], the $q$-space formalism was introduced by Callaghan [8] and Cory [9]. They demonstrated that the Fourier transform of the normalized signal decay $E(\mathbf{q})$, with respect to the diffusion wave-vector $\mathbf{q}=(2 \pi)^{-1} \gamma \delta \mathbf{g}$ can be related to the displacement probability function (PDF) $P$, as

$$
P(\mathbf{p})=\int_{\mathbf{q} \in \mathbb{R}^{3}} E(\mathbf{q}) \exp \left(-2 \pi i \mathbf{q}^{\mathrm{T}} \mathbf{p}\right) d \mathbf{q},
$$

where $\mathbf{p} \in \mathbb{R}^{3}$ stands for the displacement vector. Note that the DTI method can be described in this formalism with $E(\mathbf{q})=$ $\exp \left(-4 \pi^{2} \Delta \mathbf{q}^{\mathrm{T}} D \mathbf{q}\right)$ [10]. Eq. (2) shows that under the assumption of a narrow pulse approximation ( $\delta$ very short and $\delta \ll \Delta$ ), the PDF can be retrieved by a Fourier transform of the pseudoperiodic diffusion signal. Eq.(2) leads to a method known as Diffusion Spectrum Imaging (DSI) [11] which is not restricted to a particular diffusion model in contrast to DTI. It is theoretically one of the most promising method to recover complex information from the diffusion data. However it is not clini- 
cal compliant since it requires a very high magnetic field and a huge number of acquisitions to sample the whole $q$-space coefficients meanwhile the patient must stay motionless. To overcome these problems, two alternative subsets of methods have been developed in the literature. They focus on the measure of either radial or angular features of the PDF.

$q$-space Nuclear Magnetic Resonance (NMR) provides a radial analysis of the MR signal intensity. It was first introduced by Tanner et al. [12, 13]. These studies are highly related to DWI Eq.(1) with the main difference that the latter allows to measure the signal along multiple $b$ values. $q$-space NMR experiments provides clues of the radial diffusion behavior of solvents and solutes in heterogeneous systems, including yeast cells, human erythrocytes and tissues [9, 14, 15]. Additionally, $q$-space NMR enables to validate complex diffusion models such as the impermeable parallel planes method [16, 17]. This model aims to better understand the origin of the diffusion MR signal under the assumption that the signal peaks are related to the barriers that restrict the diffusion [8]. However this method focuses only on the radial reconstruction of the MR signal and do not recover angular information. Yet, several promising recent studies $[18,19]$ propose new echo sequences optimized for $q$-space NMR.

High Angular Resolution Diffusion Imaging (HARDI) introduced by Tuch et al. in [20] has allowed the angular analysis of the MR signal intensity. This sampling protocol proposes to acquire the diffusion signal reduced to a single sphere in the $q$-space. Few methods have been proposed so far to compute the whole PDF or some angular features of it. From a quite limited (25-100) number of samples, Tuch introduced in [21] the Q-Ball Imaging (QBI) technique. It computes the Orientation Density Function (ODF) which is the radial projection of the PDF modeled as a spherical function able to represent crossing fibers. The estimation of the ODF is commonly used as a pre-process to fiber-tracking in the human brain white matter $[22,23]$. The ODF computation involves the Funk-Radon transform (FRT) which corresponds to the Fourier Transform on the sphere. However the result is a convolution of the true ODF with a Bessel function so that each direction undesirably get corrupted by neighbor directions. The Fiber Orientation Distribution (FOD) method and its derivatives [6, 24, 25] try to compute the whole PDF volume by the deconvolution of the diffusion signal. The considered deconvolution kernel usually represents the signal of a single fiber model and requires a prior on either angular or radial MR signal or both. The Diffusion Orientation Transform (DOT) [26] method assumes a monoexponential radial MR decay and proposes analytical solutions to the Fourier transform using the Hankel transform. Nonetheless, the HARDI technique reduces the number of samples and the acquisition time, by restricting the acquisition to be on a single sphere. Consequently all HARDI-based methods assume strong priors on the radial behavior of the MR signal; and thus an inaccurate radial estimation of the MR signal may lead to a corrupted estimation of angular features of the PDF, because of the duality between the $q$-space and the probability space.

Multi-sphere HARDI: In order to overcome the problems of the long acquisition time (as in DSI) and the missing radial or angular sampling (as in HARDI and $q$-space NMR), Hybrid Diffusion Imaging (HYDI) has been recently introduced by $\mathrm{Wu}$ et al. in [27]. It proposes to extend the acquisition to multiple spheres in the $q$-space. Since the number of samples on a sphere varies along its radius, it does not necessarily mean that HYDI needs more samples than HARDI. Indeed, whereas HARDI focuses on having a lot of samples on a single sphere, HYDI focuses on a better distribution of samples on the $q$ space. Very few methods have been proposed so far to take advantage of this kind of sampling: the generalized DTI (GDTI) method [28, 29] is based on the Fick's diffusion law, and the DOT method $[26,30]$ has been extended to multi-exponential radial decay. Nonetheless these methods use a larger set of data and are still based on prior models of the input signal radial behavior. Khachaturian et al. [31] proposed to sample the MR signal on two spheres of the $q$-space, to estimate the ODF independently for each sphere and non-linearly merge them in the spherical wavelet domain. Yet the approach relies on an empiric maxmod merging function. Recently, Pickalov et al. [32] used a tomographic scheme with prior on the PDF and the MR signal to reconstruct the whole PDF. Nevertheless, the method still requires a large set of data samples (approximately 500).

Robustness to MRI noise: Besides, MRI data are acquired as complex values corrupted with thermal noise and skin depth effect $[33,34]$. The amplitude of this noise is well characterized by a Gaussian probability density function in the real and imaginary parts of the complex data [35]. The scanner only provides the magnitude of the complex data, so the initial noise in complex space is non-linearly transformed into Rician-distributed noise [36, 37, 38]. When the Signal-to-Noise Ratio (SNR) is high, it is known that the Rician distribution can be conveniently approximated by a Gaussian distribution. However, Rician noise induces a bias in the MR intensities especially for lower SNR, i.e. at high $q$ values, and undesirably leads to overestimated diffusion measures [36]. Several studies has tackled this issue [39, 40, 41, 42, 43], but these methods are restricted to HARDI acquisitions. To the extend of our knowledge, no appropriate methods have been proposed for the robust estimation of dMRI signal from a HYDI-like dataset so far.

In this paper, we present a flexible and robust method to compute various features on the PDF. The proposed approach is based on a "model-free" continuous reconstruction of the MR signal from a HYDI-like dataset (section 2). Since in-vivo data are corrupted by acquisition noise, we also propose a variational variant of the estimation method, taking the Rician noise property into account (section 3 ). We finally illustrate our results on both numerical and real human datasets (section 4).

\section{Estimation of PDF-based features}

In this section, we focus on the estimation of PDF features at a single voxel of a HYDI-like dataset. Since the MR signal is under-sampled in the $q$-space, the direct reconstruction of the PDF (or a PDF feature) would raise numerical stability and precision issues. Instead, we propose to reconstruct the signal as a continuous function. Section 2.1 introduces an orthonormal basis based on a series of Gaussian-Laguerre and spher- 


\begin{tabular}{|l|l||l|l|}
\hline Symbol & Description & Symbol & Description \\
\hline \hline PDF & Probability Density Function & $\mathbf{p}, \mathbf{k}$ & Water displacement vectors in $\mathbb{R}^{3}$ \\
ODF & Orientation Density Function & $\mathbf{q}$ & Diffusion space wave-vector in $\mathbb{R}^{3}$ \\
FRT & Funk-Radon Transform & $P(\mathbf{p})$ & Average displacement PDF at $\mathbf{p}$ \\
SH & Spherical Harmonics & $\mathcal{G}(\mathbf{k})$ & PDF feature at point $\mathbf{k}$ \\
SPF & Spherical Polar Fourier & $H_{\mathbf{k}}(\mathbf{p})$ & Projection function of $\mathcal{G}(\mathbf{k})$ \\
$a_{n, l, m}$ & SPF expansion coefficient at order $n, l$ and $m$ & $S(\mathbf{q})$ & MR signal at diffusion gradient $\mathbf{q}$ \\
$E(\mathbf{q})$ & Normalized MR signal $S(\mathbf{q}) / S(0)$ & $\mathbf{E}_{i}$ & $i$-th sample of the discrete signal vector $\mathbf{E}$ \\
$\hat{E}(\mathbf{q})$ & Reconstructed signal at $\mathbf{q}$ & $\hat{\mathbf{E}}_{i}$ & $i$-th sample of the reconstructed discrete signal vector $\hat{\mathbf{E}}$ \\
$n_{s}$ & Number of MR signal samples & $n_{c}$ & Number of SPF coefficients \\
\hline
\end{tabular}

Table 1: A list of major notations used in this paper.

ical harmonics functions in which we expect the signal to be sparse. Indeed, this basis exhibits mono-exponential radial decay when it comes to low-order truncation. Once the continuous approximation of the MR signal is reconstructed, we propose in section 2.2 a straightforward method to extract various features that can be expressed as projection functions. We also provide a computational optimization for spherical features only (section 2.3). For convenience, a list of notations commonly used in this paper is reminded in Table 1.

\subsection{Spherical Polar Fourier Expansion}

In the following, we present the use of an adapted series to reconstruct the continuous signal from the MR data samples. To take advantage of whatever the acquisition protocol is (generally one or several spheres in the $q$-space), we define an orthonormal basis from a combination of angular and radial elementary functions expressed in spherical coordinates. Then, we expand the MR signal attenuation $E$ as the following series in the orthonormal basis Spherical Polar Fourier (SPF):

$$
E(\mathbf{q})=\frac{S(\mathbf{q})}{S(0)}=\sum_{n=0}^{\infty} \sum_{l=0}^{\infty} \sum_{m=-l}^{l} a_{n, l, m} R_{n}(\|\mathbf{q}\|) y_{l}^{m}\left(\frac{\mathbf{q}}{\|\mathbf{q}\|}\right)
$$

so that $n \in \mathbb{N}$ is the radial index, and $l \in \mathbb{N}, m \in \mathbb{Z},-l \leq m \leq l$ are the angular indexes. $S(\mathbf{q})$ denote the diffusion MR signal at a vector $\mathbf{q}$ in the $q$-space. The symbols $a_{n, l, m}$ are the series coefficients, $y_{l}^{m}$ are the real spherical harmonics $(\mathrm{SH})$, and $R_{n}$ is an orthonormal radial basis function made of GaussianLaguerre (GL) functions. This basis is derived from the Gaussian Type Orbital functions (GTO) used in the crystallography community for the computation of the molecular electron orbitals and molecular docking $[44,45,46]$. Though the purposes are different, the used mathematical tools are similar and the use of the SPF basis enables to obtain a method of signal reconstruction independently of the acquisition protocol. In practice, the reconstructed signal $\hat{E}$ is estimated as:

$$
\hat{E}(\mathbf{q})=\sum_{n=0}^{N} \sum_{l=0}^{L} \sum_{m=-l}^{l} a_{n, l, m} R_{n}(\|\mathbf{q}\|) y_{l}^{m}\left(\frac{\mathbf{q}}{\|\mathbf{q}\|}\right),
$$

where the symbols $N$ and $L$ respectively denotes the radial and the angular truncation orders.
The angular component of the signal is reconstructed by elementary angular functions based on the complex Spherical Harmonics (SH) basis $Y_{l}^{m}$,

$$
\begin{array}{r}
Y_{l}^{m}(\theta, \phi)=\left[\frac{2 l+1}{4 \pi} \frac{(l-m) !}{(l+m) !}\right]^{1 / 2} P_{l}^{m}(\cos (\theta)) e^{i m \phi} \\
\text { with }(\theta, \phi)=\frac{\mathbf{q}}{\|\mathbf{q}\|} \in \mathcal{S}^{2}
\end{array}
$$

where $P_{l}^{m}$ are the associated Legendre polynomials. The complex coefficients SH $Y_{l}^{m}$ form an orthonormal basis for functions defined on the unit sphere and are the angular part of a set of solutions to Laplace's equation diffusion equation in spherical coordinates [47]. For this reason they have been widely used in dMRI [7, 48], especially with the following subset of the complex basis made of real and symmetric SH $y_{l}^{m}$ :

$$
y_{l}^{m}=\left\{\begin{array}{llc}
\sqrt{2} \operatorname{Re}\left(Y_{l}^{m}\right) & \text { if } & 0<m \leq l \\
Y_{l}^{0}, & \text { if } & m=0 \\
\sqrt{2} \operatorname{Im}\left(Y_{l}^{|m|}\right) & \text { if } & -l \leq m<0
\end{array} \quad \text { with } l \in 2 \mathbb{Z}\right.
$$

Indeed, as the diffusion signal exhibits real and symmetry properties, the use of the $y_{l}^{m}$ strengthens the robustness of the reconstruction to signal noise and reduces the number of required coefficients [7]. As a result, whereas a complex spherical harmonics series expansion would involve $(L+1)^{2}$ coefficients, a real spherical harmonics series expansion to order $l \leq L$ involves only $(L+1)(L+2) / 2$ coefficients.

The radial component of the MR signal is reconstructed by the functions $R_{n}$. Since the number of radial samples is highly limited, we expect the radial signal to be sparse in $R_{n}$, i.e. to capture the radial attenuation of $E$ with a few orders $n$. Several studies $[49,50,51,52,53]$ have reported that the signal decay seems to be a composition of Gaussian functions, the number of functions being determined by the wave-vector norm $\|\mathbf{q}\|$. Based on experimental observations, some studies have proposed to model this local diffusion using a bi-exponential function [51, 52], suggesting a slow and a fast free diffusion in correspondence to the intra and extra cellular compartments. Indeed, contrary to anatomical MRI, diffusion MRI has a low spatial resolution and consequently a voxel size of $2-3 \mathrm{~mm}$ represents a local average diffusion. The spatial resolution of voxels is such that it forms a complex physical system which 
contains numerous cells with various features. Therefore, a direct relationship between the bi-exponential attenuation and the intra-extra cellular diffusion is not obvious and has yet to be investigated [51]. It is still unclear how the bi-exponential model accurately relates to the real diffusion behavior [50]. In this paper, we propose a general estimation of the radial part of the signal attenuation $E$ using a normalized basis of generalized Gaussian-Laguerre polynomials $R_{n}$ :

$$
\begin{aligned}
R_{n}(\|\mathbf{q}\|) & =\left[\frac{2}{\zeta^{3 / 2}} \frac{n !}{\Gamma(n+3 / 2)}\right]^{1 / 2} \exp \left(-\frac{\|\mathbf{q}\|^{2}}{2 \zeta}\right) L_{n}^{1 / 2}\left(\frac{\|\mathbf{q}\|^{2}}{\zeta}\right), \\
L_{n}^{(\alpha)}(x) & =\left((\alpha+1)_{n} / n !\right) 1 F 1(-n, \alpha+1, x)
\end{aligned}
$$

where $\zeta$ denotes the scale factor, $L_{n}^{(\alpha)}(x)$ are the generalized Laguerre polynomials which are the eigenfunctions of the FourierBessel transform [54]. The notation $(a)_{n}$ denotes the Pochhammer symbol and $1 F 1$ denotes the confluent hyper-geometric function. Refer to appendix A for simplified expression of Eq.(8) and to Fig.1b for a plot of $R_{n}$. A radial truncation order $n \leq N$ involves $(N+1)$ coefficients. A low order $N$ assumes a radial Gaussian behavior as in [26, 30]; the Gaussian decay in the SPF basis arises from the normalization of the Laguerre polynomials in the spherical coordinates (more details in appendix B). On the contrary, a high order $N$ provides a modelfree estimation.

Note that the truncation orders $N$ and $L$ are directly related to the number of data samples in the acquisition, and conveniently adjusts the trade-off between the number of samples and the prior on the MR signal. In practice, $N$ and $L$ are chosen so that the number of coefficients $n_{c}=(N+1)(L+1)(L+2) / 2$ is roughly the half of the number of samples $n_{s}$, i.e. $(N+1)(L+$ $1)(L+2) \geq n_{s}$. The choice to favour $N$ or $L$ depends widely on the sampling distribution of the $q$-space and eventually on the feature to observe: large $L$ for angular features (such as anisotropy maps, fibertracking), or large $N$ for radial features (such as number of compartiment, axon measurement).

It is worth to note that Özarslan et al. [55] have proposed a quite similar method for the reconstruction of MR radial only signals ( $q$-space NMR). Their basis is based on the Hermite polynomials which are the eigenfunctions of the Fourier transform. They can be analytically related to Eq. (8) (see Appendix E for more details). Additionally, the QBI [21, 40, 56] and the DOT [26] methods can be expressed in our approach with respectively $R_{n}(\|\mathbf{q}\|)=\delta\left(\|\mathbf{q}\|-q^{\prime}\right) / q^{\prime 2}$ and $R_{n}(\|\mathbf{q}\|)=$ $j_{n}\left(2 \pi\|\mathbf{q}\| R_{0}\right) \delta_{n, l}$ where $j_{n}$ is the spherical Bessel function at order $n, q^{\prime}$ and $R_{0}$ are two real constants (appendix C and D for more details on this).

Fig. 1 points out the actual adequacy of the first $R_{n}$ functions to the experimental MR signal from erythrocytes (appendix A for more details). This experiment involves a bi-homogeneous unrestricted diffusion for decreasing values of the hematocrit, i.e. the proportion of red blood cells within blood. The diffusion decay is of course expected to be more complex in the human brain white matter, it is clear that our basis seems to be well adapted to model complex diffusion profiles. The scale factor $\zeta$ can be easily estimated from experimental values of decay on data samples. We propose an empiric formula in Eq.(24).
Spherical Polar Fourier (SPF) is a set of functions which forms a complete, orthogonal basis,

$$
\int_{\mathbf{q} \in \mathbb{R}^{3}}\left[R_{n}(\|\mathbf{q}\|) y_{l}^{m}\left(\frac{\mathbf{q}}{\|\mathbf{q}\|}\right)\right]\left[R_{n^{\prime}}(\|\mathbf{q}\|) y_{l^{\prime}}^{m^{\prime}}\left(\frac{\mathbf{q}}{\|\mathbf{q}\|}\right)\right] d \mathbf{q}=\delta_{n n^{\prime}} \delta_{l l^{\prime}} \delta_{m m^{\prime}}
$$

The square error between a function and its expansion converges to zero as the truncation orders $N$ and $L$ become infinite.

For each voxel, we propose to fit our SPF basis to the $q$ space signal using a damped least square minimization technique. Best fitting coefficients $a_{n, l, m}$ are given by the regularized Moore-Penrose pseudo-inverse scheme:

$$
\begin{gathered}
\mathbf{M}=\left[\begin{array}{ccc}
R_{0}\left(\left\|\mathbf{q}_{1}\right\|\right) y_{0}^{0}\left(\frac{\mathbf{q}_{1}}{\left\|\mathbf{q}_{1}\right\|}\right) & \ldots & R_{N}\left(\left\|\mathbf{q}_{1}\right\|\right) y_{L}^{L}\left(\frac{\mathbf{q}_{1}}{\left\|\mathbf{q}_{1}\right\|}\right) \\
\vdots & \ddots & \vdots \\
R_{0}\left(\left\|\mathbf{q}_{n_{s}}\right\|\right) y_{0}^{0}\left(\frac{\mathbf{q}_{n s}}{\left\|\mathbf{q}_{n_{s}}\right\|}\right) & \ldots & R_{N}\left(\left\|\mathbf{q}_{n_{s}}\right\|\right) y_{L}^{L}\left(\frac{\mathbf{q}_{n_{s}}}{\left\|\mathbf{q}_{n_{s}}\right\|}\right)
\end{array}\right] \\
\mathbf{E}=\left(E\left[\mathbf{q}_{1}\right], \ldots, E\left[\mathbf{q}_{n s}\right]\right)^{\mathrm{T}}, \quad \mathbf{A}=\left(a_{000}, \ldots, a_{N L L}\right)^{\mathrm{T}} \\
\mathbf{A}=\underset{\mathbf{A} \in \mathbb{R}^{n_{c}}}{\arg \min }\|\mathbf{E}-\mathbf{M} \mathbf{A}\|^{2}+\lambda_{l}\|\mathbf{L}\|^{2}+\lambda_{n}\|\mathbf{N}\|^{2} \\
=\left(\mathbf{M}^{\mathrm{T}} \mathbf{M}+\lambda_{l} \mathbf{L}^{\mathrm{T}} \mathbf{L}+\lambda_{n} \mathbf{N}^{\mathrm{T}} \mathbf{N}\right)^{-1} \mathbf{M}^{\mathrm{T}} \mathbf{E}
\end{gathered}
$$

where $n_{s}$ is the number of data samples, $\mathbf{M}$ denotes the SPF basis matrix and $\mathbf{E}, \mathbf{A}$ respectively denote the vectors of data samples and the coefficients of the reconstructed signal in SPF basis. Since the matrix $\mathbf{M}$ is likely to be ill-conditioned because of the highly reduced number of samples, we introduce the regularization matrices $\mathbf{L}$ and $\mathbf{N}$ with respective entries $l(l+1)$ and $n(n+1)$ along their diagonals. These matrices penalize higher frequencies of the angular and radial parts of the SPF estimation, since we assume that they are likely to capture noise. The $\lambda_{l}$ and $\lambda_{n}$ set the angular and radial weights of the regularization. Similarly to [57], angular regularization is equivalent to the application of a smoothing filter in the angular $q$-space domain. This comes from the duality between the convolution in the $q$-space and the multiplication in the spherical harmonics domain [58]. Radial frequency regularization is equivalent to favour a mono-exponential decay.

The diffusion is a positive process, so theoretically the reconstructed signal $\hat{E}$ expressed in the SPF basis should be positive, i.e. $\hat{E}(\mathbf{q}) \geq 0, \mathbf{q} \in \mathbb{R}^{3}$. Note that Eq.(12) gives no guarantee that the reconstructed signal remains positive. However in practice, we observe that the first negative occurrence of $\hat{E}$ happens for large $\|\mathbf{q}\|$, i.e. when the original decreasing function $E$ is almost vanishing (less than $1 \%$ of its maximum value $E(0)$, more details in section 4.1). The global minimum value actually corresponds to $2 \%$ of the signal maximum amplitude. Furthermore, $\hat{E}$ converges to $E$ (i.e. 0 ) when $\|\mathbf{q}\|$ tends toward infinity. All this means that the reconstructed signal can be considered as close enough to the true one so that a simple value cut in $[0, \infty]$ is as an acceptable way of ensuring the desired positiveness constraint. 


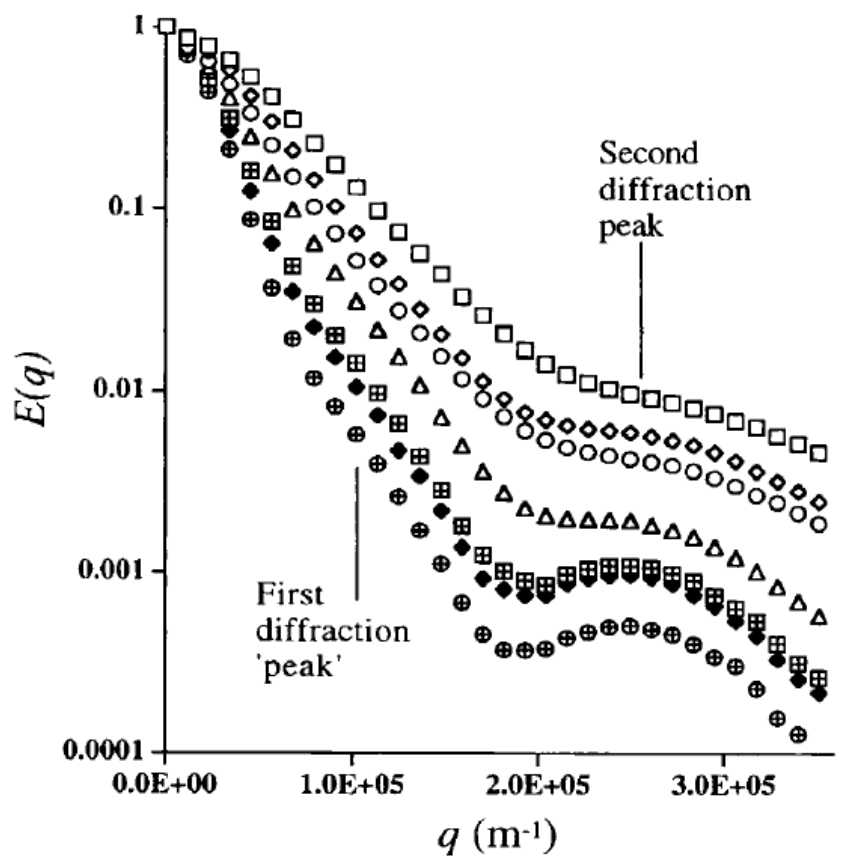

(a) Diffusion signal decay curves for water in suspensions of human erythrocytes as a function of $\|\mathbf{q}\|$ value at different hematocrit. The hematocrit values are in decreasing order from the top of the figure, starting from a value of $93 \%$, followed by $83,73,63,47,42$ and ending at $25 \%$ at the bottom of the figure (from Kuchel et al. [14])

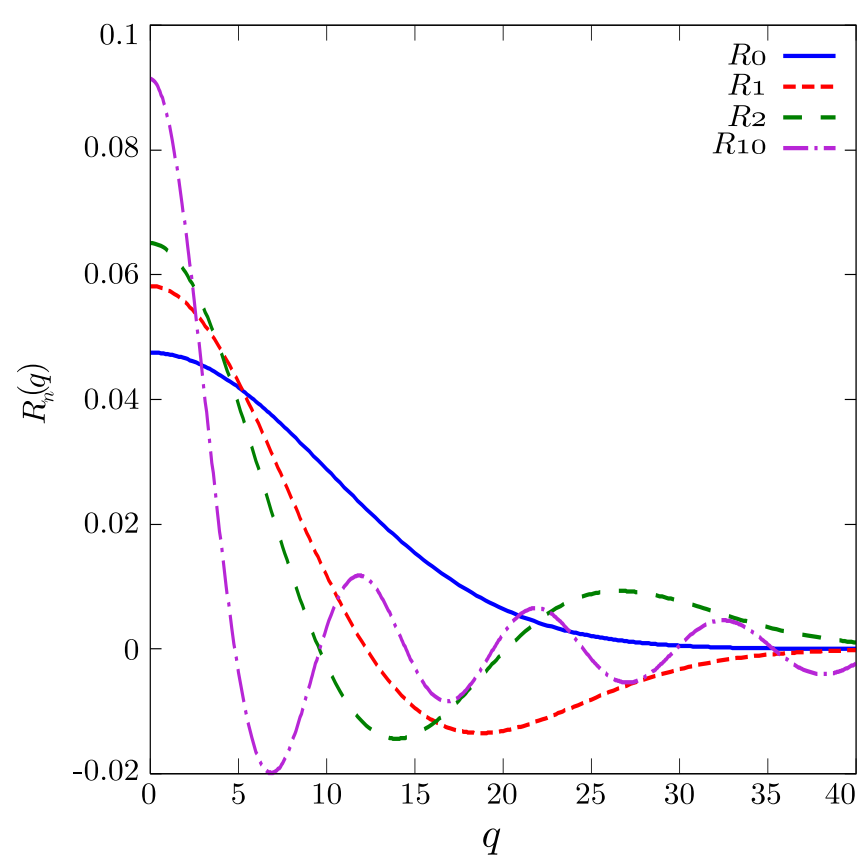

(b) First basis functions of the radial orthonormal basis $R_{n}$ with scaling factor $\zeta=100$. Low order $n$ functions exhibits Gaussian attenuation behavior whereas higher order $n$ are helpful to capture oscillating components of the MR signal.

Figure 1: Comparison of the proposed reconstruction radial basis (b) and an experimental plot of MR signal decay (a).

The condition number $C$ is a measure of how numerically well-conditioned the regularized matrix $\mathbf{M}_{r e g}=\mathbf{M}^{\mathrm{T}} \mathbf{M}+$ $\lambda_{n} \mathbf{N}^{\mathrm{T}} \mathbf{N}+\lambda_{l} \mathbf{L}^{\mathrm{T}} \mathbf{L}$ is

$$
C=\left\|\mathbf{M}_{\text {reg }}\right\|_{\infty}\left\|\mathbf{M}_{\text {reg }}^{-1}\right\|_{\infty}
$$

Therefore the parameters $\lambda_{n}$ and $\lambda_{l}$ should be chosen so that the condition number of the matrix $\mathbf{M}_{\text {reg }}$ is not too large, typically $C \approx 10^{4}$ according to our experiments.

\subsection{Features of the PDF}

Now, we have a continuous representation of the signal $\hat{E}$ from the SPF coefficients. Let $\mathcal{G}(\mathbf{k})$ be a feature of the PDF at point $\mathbf{k}$, expressed as:

$$
\mathcal{G}(\mathbf{k})=\int_{p \in \mathbb{R}^{3}} P(\mathbf{p}) H_{\mathbf{k}}(\mathbf{p}) d \mathbf{p}
$$

where $H_{\mathbf{k}}$ denotes a projection function at point $\mathbf{k}$. Table 2 represents several popular features $\mathcal{G}$ which can be evaluated using our computational scheme. A naive way to retrieve $\mathcal{G}$ would be to reconstruct $E$ from the SPF coefficients, compute a Fast Fourier Transform (FFT) to get the complete PDF and finally calculate $\mathcal{G}$ on the estimated PDF volume; however such a scheme would induce cumbersome computations which would raise numerical accuracy issues. Instead, we propose to compute $\mathcal{G}(k)$ directly from the SPF coefficients. Indeed, since the SPF are an orthonormal basis the following relation holds:

$$
\mathcal{G}(\mathbf{k})=\int_{\mathbf{p} \in \mathbb{R}^{3}} P(\mathbf{p}) H_{\mathbf{k}}(\mathbf{p}) d \mathbf{p}=\int_{\mathbf{q} \in \mathbb{R}^{3}} E(\mathbf{q}) h_{\mathbf{k}}(\mathbf{q}) d \mathbf{q}=\sum_{n, l, m}^{\infty} a_{n, l, m} b_{n, l, m}^{\mathbf{k}}
$$

where $h_{\mathbf{k}}$ is the inverse Fourier transform of $H_{\mathbf{k}}$ and $a_{n, l, m}, b_{n, l, m}^{\mathbf{k}}$ respectively denote the SPF expansion of $E$ and $h_{\mathbf{k}}$. Therefore, the computation of $\mathcal{G}(\mathbf{k})$ from Eq. (15) simply turns into a very fast dot product between two vectors of SPF coefficients. YuChien et al. proposed a similar approach in [27] where some specific features of the PDF are computed by a projection in the $q$-space between a feature function $h$ and diffusion signal $E$. However, the latter method is not based on a continuous representation of the signal, and numerical issues coming from the computation of the integral $\int_{\mathbf{q}} E(\mathbf{q}) h(\mathbf{q}) d \mathbf{q}$ with a discrete sampling arise. Note that with our settings, the QBI and the DOT methods analytically express themselves as specific features of the PDF, respectively the FRT and the iso-radius.

\subsection{Optimization for angular features}

At this point, we have to compute $H_{\mathbf{k}}, h_{\mathbf{k}}$ and $b_{n, l, m}^{\mathbf{k}}$ for each probability vector $\mathbf{k} \in \mathbb{R}^{3}$. Actually, all these computations are required only once and can be stored in computer memory for several use since they are data-independent. Nonetheless, an alternative faster and memory-saving scheme arises for features which are angular-dependent only; i.e. $\mathbf{k} \in \mathcal{S}^{2}$ where $\mathcal{S}^{2}$ is the unit sphere domain. Note that features $\mathcal{G}$ as defined in table 2 satisfy this constraint. In this case, we use the rotation property of spherical harmonics which states that a rotation of a spherical harmonic can be computed as a linear combination of spherical harmonics of the same degree [58]. Therefore, under a rotation transform expressed in terms of Euler angles $(\alpha, \beta, \gamma)$, any rotated SPF can be expressed as a linear combination of SPF of 


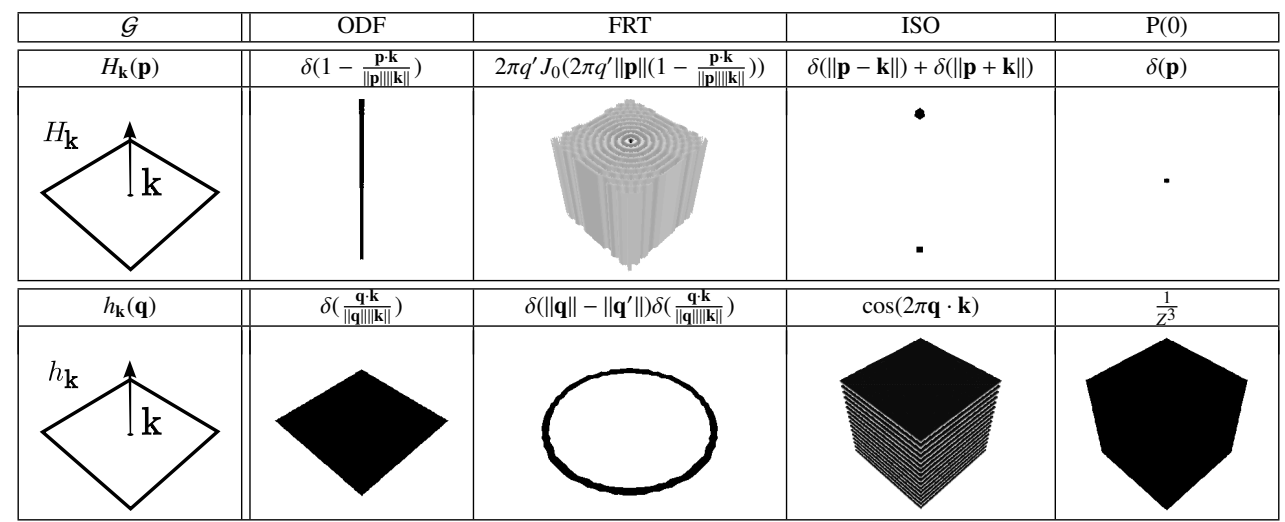

Table 2: A non-exhaustive list of some PDF features $\mathcal{G}$ and their projection function $H_{\mathbf{k}}$ at point $\mathbf{k}$. This features are computed as volumic image of size $Z^{3}=64^{3}$ voxels and shown below each formula. So given a direction $\mathbf{k}$, it illustrates how the PDF features and its expression in the $q$-space respectively looks like. The first two figures of the first column (line 2 and 4 ) indicates the direction of $\mathbf{k}$ in $\mathbb{R}^{3}$. FRT stands for the Funk-Radon Transform used in QBI, where $J_{0}$ is the Bessel function of the first kind and $\left\|\mathbf{q}^{\prime}\right\|$ is the radius of the q-ball sphere. ISO stands for isoprobability profiles. $\mathrm{P}(0)$ stands for the probability of non-displacement.

the same order:

$$
a_{n, l, m}^{\prime}=\operatorname{Rot}_{\alpha, \beta, \gamma}\left[a_{n, l, m}\right]=\sum_{m^{\prime}=-l}^{l} a_{n, l, m^{\prime}} D_{m^{\prime} m}^{(l)}(\alpha, \beta, \gamma),
$$

where $a_{n, l, m}$ and $a_{n, l, m}^{\prime}$ are respectively the coefficients of the original SPF and its rotation. $D_{m^{\prime} m}^{(l)}$ stands for the real Wigner rotation matrix expressed in terms of the Euler angles $(\alpha, \beta, \gamma)$ in the $z y z$ convention [59] (refer to appendix F for more details).

In practice, as we want to compute the projection of the PDF feature on a unit sphere, we set $(\alpha, \beta, \gamma)=(\phi, \theta, 0)$, where $\phi \in$ $[0,2 \pi)$ and $\theta \in[0, \pi]$ follow spherical coordinates from physics convention. In this case, $H_{\mathbf{k}}$ has to be constructed only once with $\mathbf{k}=(\theta, \phi)=(0,0)$, i.e. $\mathbf{k}$ initially taken as the $z$-axis unitary vector $\mathbf{z}$. We provide an overview of the proposed method in Fig.2. A step by step algorithm for angular features defined on the unit sphere $\mathcal{S}^{2}$ is detailed in the following.
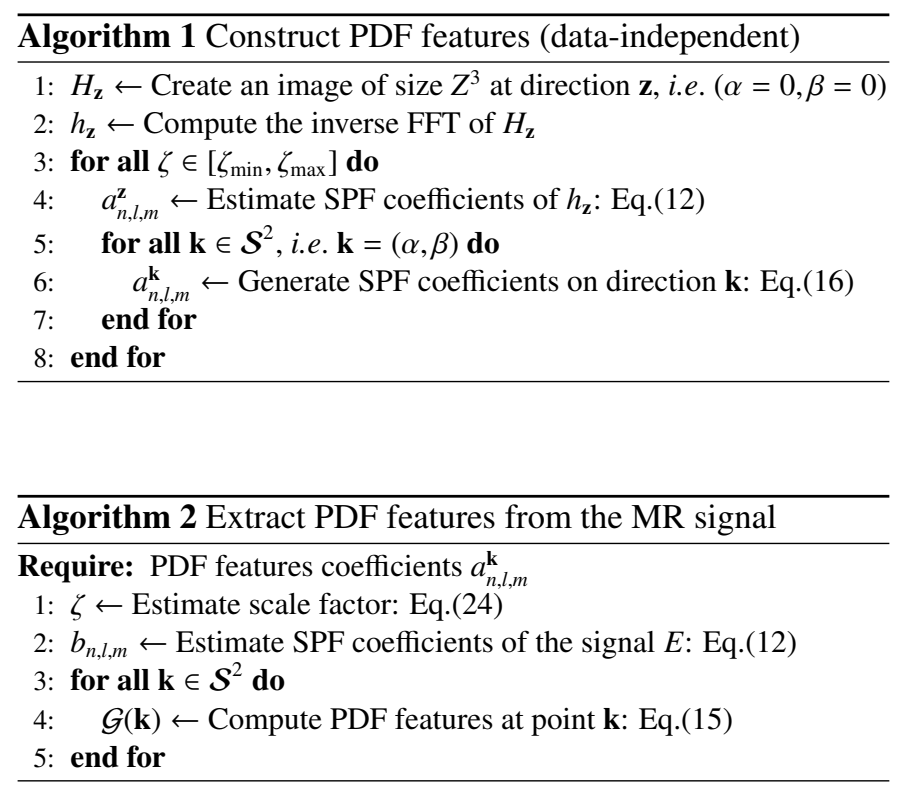

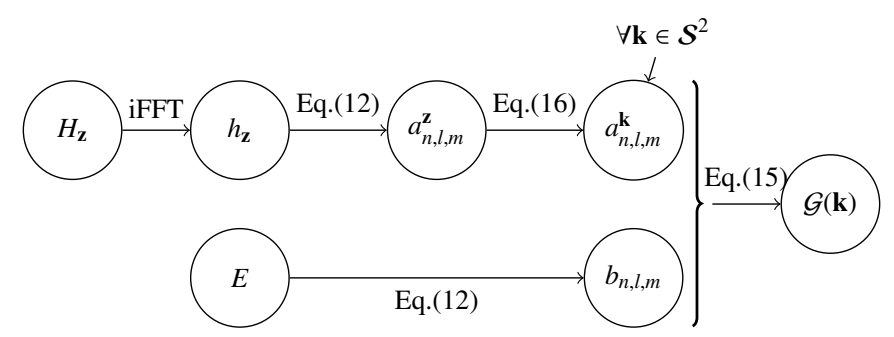

Figure 2: Algorithm overview for the computation of PDF features $\mathcal{G}$ at point $\mathbf{k}$, defined on the unit sphere $\mathcal{S}^{2}$. iFFT stands for the inverse Fast Fourier Transform.

\section{Adding robustness to Rician noise}

It is known that MRI data are acquired as complex values and corrupted by white noise. This noise can well be characterized by a Gaussian PDF in the real and imaginary part. In practice, magnitude images are traditionally used as there are insensitive to phase shifts artifacts (such as chemical shifts of inhomogeneities) [37].

It is common practice to assume that the noise in the magnitude images follows a Gaussian PDF. However, several studies have shown that the noise is Rician distributed [36, 60], which reduce to a Rayleigh distribution in area with no MR signal [61]. The Rician noise induces a bias in the MR intensities, which on average leads to overestimated values.

Since the acquisition noise on the MR signal is not Gaussian, a least square fit (12) is definitely not the best choice for the data estimation process. This issue arises especially when dealing with low SNR data as this is the case for very high $q$ values. Furthermore, independent voxel estimation does not reflect the spatial regularity of the diffusion function. We propose to tackle these issues with a variational framework which is adaptable to noise distribution and is able to use valuable information provided by nearby voxels. We focus now on the estimation of PDF features of a entire volume of voxels from a noisy dMRI dataset. 


\subsection{Variational Formulation}

The key idea is to estimate and regularize the whole volume of PDF features at the same time. This enables to take into account correlation between all local data samples of the processing pipeline instead of doing the different parts separately. Let $\Omega \subset \mathbb{R}^{3}$ be the domain of datasets voxels $\mathbf{x}$ and $\mathbf{E}: \Omega \rightarrow \mathbb{R}^{n_{s}}$ the acquired dMRI volume at voxel $\mathbf{x}$ corrupted by Rician noise. We seek the SPF coefficients $\mathbf{A}: \Omega \rightarrow \mathbb{R}^{n_{c}}$ of the filtered dMRI volume at voxel $\mathbf{x}$ so that $\hat{\mathbf{E}}=\mathbf{M A}$, where the symbol $\mathbf{M}=\left(R_{n}\left(\left\|\mathbf{q}_{j}\right\|\right) y_{l}^{m}\left(\frac{\mathbf{q}_{j}}{\left\|\mathbf{q}_{j}\right\|}\right)\right)_{(n, l, m \times j) \in\left(n_{c} \times n_{s}\right)}$ denotes the SPF basis matrix Eq.(12). We propose to estimate and regularize the SPF coefficients field from the dataset volume simultaneously by minimizing the following nonlinear functional energy:

$$
\mathbf{A}=\underset{\mathbf{A} \in \mathbb{R}^{n_{c}}}{\arg \min }\left\{\int_{\mathbf{x} \in \Omega}\left[\sum_{i=0}^{n_{s}} \psi\left(\hat{\mathbf{E}}_{i}\right)\right]+\alpha_{r} \varphi(\|\nabla \mathbf{A}\|) d \Omega\right\}
$$

The likelihood term $\psi\left(\hat{\mathbf{E}}_{i}\right)$ measures the dissimilarities at voxel $\mathbf{x} \in \Omega$ between $\mathbf{E}$ and its reconstruction $\hat{\mathbf{E}}$ for the $i$-th sample. Let $\psi: \mathbb{R} \rightarrow \mathbb{R}$ and $\varphi: \mathbb{R} \rightarrow \mathbb{R}$ are both real functions, $\alpha_{r} \in \mathbb{R}$ is the regularization weight and $\|\nabla \mathbf{A}\|$ the gradient norm defined as

$$
\|\nabla \mathbf{A}\|=\sum_{n=0}^{\infty} \sum_{l=0}^{\infty} \sum_{m=-l}^{l}\left\|\nabla \mathbf{A}_{n, l, m}\right\|
$$

Note that if $\psi(s)=s^{2}$ and $\alpha_{r}=0$ in Eq.(17), we minimize the least square criterion given at Eq. (12). However, in the general case the minima cannot be computed straightforwardly, so we apply a gradient descent coming from the Euler-Lagrange derivation of Eq.17. It leads to the following set of multi-valued Partial Differential Equations (PDE). In practice, we first set $\mathbf{A}_{(t=0)}$ to $\mathbf{A}_{t_{0}}$, an initial estimate of the SPF coefficients. The velocity $\frac{\partial \mathbf{A}}{\partial t}$ gives the direction from the current $\mathbf{A}_{t}$ to the next $\mathbf{A}_{t+1}$ until the nearest minimum is computed.

$$
\begin{cases}\mathbf{A}_{t=0} & =\mathbf{A}_{t_{0}} \\ \frac{\partial \mathbf{A}_{j}}{\partial t} & =\sum_{i}^{n_{s}} \mathbf{M}_{i, j} \frac{\partial \psi}{\partial \mathbf{A}_{j}}\left(\hat{\mathbf{E}}_{i}\right)+\alpha_{r} \operatorname{div}(\varphi(\|\nabla \mathbf{A}\|))\end{cases}
$$

where $\frac{\partial \psi}{\partial \mathbf{A}_{j}}=\frac{\partial \psi}{\partial \hat{\mathbf{E}}} \frac{\partial \hat{\mathbf{E}}}{\partial \mathbf{A}_{j}}$ and $\hat{\mathbf{E}}=\mathbf{M A}$. The iterative gradient descent is then applied until convergence (typically when $\left.\varepsilon \in \mathbb{R}^{+}, \varepsilon \rightarrow 0, \frac{\partial \mathbf{A}}{\partial t}<\varepsilon\right)$, Similar methods have been proposed for the regularization of the DTI $[62,63,64,65]$, of the ADC $[66,67]$ and the ODF [43]. Yet none of these methods is able to take full advantage of the information provided by the HYDI sampling.

\subsection{Likelihood function $\psi$}

Choosing the $\psi$ function is done by considering the Rice distribution of the noise whose probability density function is:

$$
p(E \mid \hat{E}, \sigma)=\frac{E}{\sigma^{2}} \exp \left(\frac{-\left(E^{2}+\hat{E}^{2}\right)}{2 \sigma^{2}}\right) I_{0}\left(\frac{E \cdot \hat{E}}{\sigma^{2}}\right)
$$

where $\sigma$ is the standard deviation of the noise and $I_{0}$ is the modified zeroth-order Bessel function of the first kind. We adapt the Rician bias correction filter introduced in [68] from the 2nd-order DTI to the SPF basis. It is based on a maximum a posteriori approach so we construct the filtered volume $\hat{E}$ that maximizes the log-posterior probability. We are interested in the likelihood term $p(E \mid \hat{E})$, and Eq.(20) leads to the pointwise log-likelihood:

$$
\log p(E \mid \hat{E}, \sigma)=\log \frac{E}{\sigma^{2}}-\frac{\left(E^{2}+\hat{E}^{2}\right)}{2 \sigma^{2}}+\log I_{0}\left(\frac{E \cdot \hat{E}}{\sigma^{2}}\right)=\psi(\hat{E})
$$

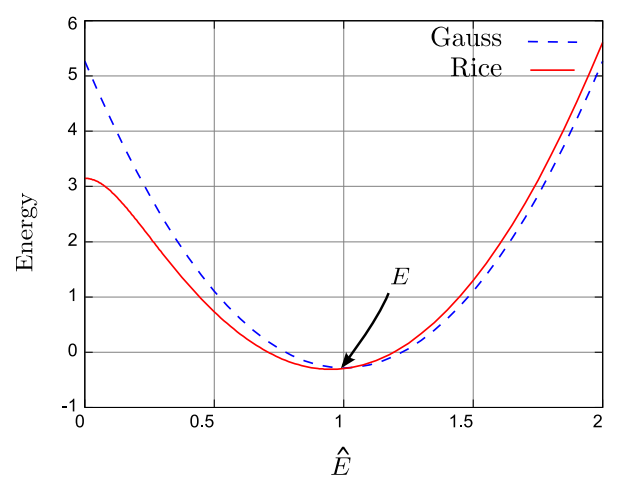

Figure 3: Energy $p(E \mid \hat{E}, \sigma)$ associated to respectively Gaussian and Rician likelihood $\psi$ functions. Note the bias introduced by the Rician function on low SNR data. $E=1$ and $\sigma=0.5$.

Fig.3 illustrates variation of the opposite function with scalar values of $\hat{E}$ when $E=1$ and $\sigma=0.5$. The energy is low when $E \approx \hat{E}$ and increases with their dissimilitudes. Note that $\sigma$ has to be known a priori and can be either retrieved from multiple images [69, 38] or a single image as described in [37, 70, 71]. Combining Eq.(19) and the derivative of Eq.(21) with respect to $\mathbf{A}_{j}$ gives the PDE that minimizes Eq.(17) and is adapted to Rician noise,

$$
\begin{aligned}
\frac{\partial \mathbf{A}_{j}}{\partial t} & =\frac{\sum_{i}^{n_{s}} \mathbf{M}_{i, j}}{\sigma^{2}}\left(-\hat{\mathbf{E}}_{i}+\mathbf{E}_{i}\left[\frac{I_{1}\left(\frac{\mathbf{E}_{i} \cdot \hat{\mathbf{E}}_{i}}{\sigma^{2}}\right)}{I_{0}\left(\frac{\mathbf{E}_{i} \cdot \hat{\mathbf{E}}_{i}}{\sigma^{2}}\right)}\right]\right) \\
& +\alpha_{r} \operatorname{div}(\varphi(\|\nabla \mathbf{A}\|))
\end{aligned}
$$

Eq.(22) relates the MR signal samples to its continuous function taking into account the specific PDF of the acquisition noise. Therefore Eq.(22) should be preferred to the least square estimation especially when dealing with low SNR quality data as the Gaussian assumption for noise is no more valid in this case.

\subsection{Regularization function $\varphi$}

The few number of samples coupled with the acquisition noise lead to a high uncertainty of the voxel signal intensity. We partially attenuate this uncertainty by combining data from voxels in a same local neighborhood, this assumption being weighted by $\alpha_{r}$ as shown in Eq.(19). We compute the distance between two voxels directly from the SPF coefficients. There are at least two advantages of this approach: it is fast to compute and it conveniently separates the distance measure in angular and radial parts $\left(\sum_{l, m}\left\|\nabla \mathbf{A}_{n, l, m}\right\|\right.$ and $\left.\sum_{n}\left\|\nabla \mathbf{A}_{n, l, m}\right\|\right)$ thanks to the SH rotational property Eq.(16) and the low-order GL Gaussian 
behavior. Note that the regularization term $\|\nabla \mathbf{E}\|=\|\nabla \mathbf{A}\|$ (more details in appendix G).

Various functions $\varphi$ from the image processing literature can be used here as long as they preserve the important features of the signal (discontinuities of the SPF coefficients). In the following experiments, we chose the hyper-surface function $1 / \sqrt{1+\|\nabla \mathbf{A}\|^{2}}$ as it is numerically stable for $\|\nabla \mathbf{A}\| \in \mathbb{R}^{3}$ and provides an anisotropic regularization behavior which fits well to the previous constraints.

\subsection{Initialization of the gradient descent}

The choice of the initial field $\mathbf{A}_{t_{0}}$ is important as it influences the quality of the signal reconstruction. Bad initialization could undesirably increases the computational time as well. A good choice requires to start from an initial field which is not so far from the global minimum. The linear LS minimization at Eq.(12) presented in section 2 seems to be an adequate alternative since it is the global minimum when $\psi(s)=s^{2}$ and $\alpha_{r}=0$. Fig. 4 illustrates that the gradient descent with $\mathbf{A}_{t_{0}}$ set to LS is smoother than choosing a random initialization $\mathbf{A}_{t_{0}}$. Thus with the former, it is possible to increase the evolution step $d t$ to bring down the number of iterations required to converge without compromising the numerical stability. Besides, the results in Fig.4b show that gradient descent with a random initialization gives a worse result (PSNR $=20.40 \mathrm{~dB}$ ) than the linear LS estimation (PSNR=26.04 dB). However, Rician estimation undoubtedly gives the best reconstruction $(\mathrm{PSNR}=33.62 \mathrm{~dB})$.

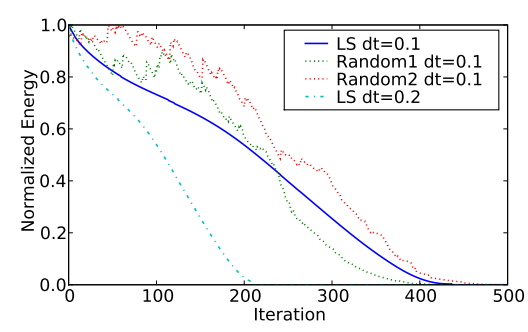

(a)

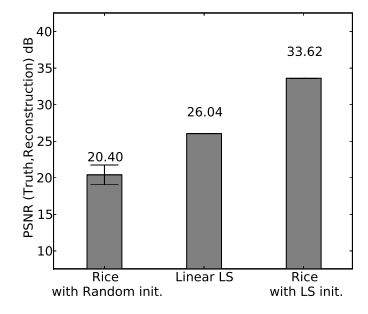

(b)
Figure 4: Influence of initial estimate $A_{0}$ on the convergence of the gradient descent. Data were noised so that the PSNR(Truth,Noisy)=19.5 dB. The Rician likelihood function refers to Eq.(22). a) Rate of convergence. Label from the top to bottom: least square initialization, random initialization 1 and 2, least square initialization with an evolution step $d t$ twice as big. b) Quality of the result. Label from the left to the right: PDE with 50 random initialization, linear LS, PDE with LS initialization.

\section{Experiments}

In this section, we present the estimation improvements along with the number of diffusion MR signal samples and demonstrate the robustness of our framework to noise. The proposed method was run on various datasets. We illustrate the results obtained on synthetic simulations (section 4.1) with both noise-free and Rician noise datasets, as well as on real human brain data (section 4.2).

\subsection{Numerical Simulations}

\subsubsection{Protocol}

We applied the above scheme to the simulations of a single fiber and crossing fiber configurations. The following synthetic multi-exponential model was used to generate the synthetic data,

$$
\left.E(\mathbf{q})=\sum_{i=1}^{N_{b}} f_{i} \exp \left(-\frac{\left(\|\mathbf{q}\|-m_{i}\right)^{2}}{\|\mathbf{q}\|^{2}} \mathbf{q}^{\mathrm{T}} \mathbf{D}_{i} \mathbf{q}\right)\right)
$$

where $\sum_{i=1}^{N_{b}} f_{i}=1$. The symbol $N_{b}$ stands for the number of fibers, $m_{i}$ is the mean diffusion and $\mathbf{D}_{i}$ is a $3 \times 3$ symmetric definite positive matrix defining the diffusion anisotropy for the $i$-th fiber. The scale factor $\zeta$ was set empirically so that the decay of the basis eigenfunctions have the same scale as the sampled data. Let $x=R_{n}\left(\left\|\mathbf{q}^{\prime}\right\|\right) / R_{n}(0)$ where $x \in[0,1]$. This leads to

$$
\zeta \approx \frac{\left\|\mathbf{q}^{\prime}\right\|^{2} \sqrt{\pi} N !}{-4 \Gamma(N+3 / 2) \log \left(x L_{N}^{1 / 2}(0)\right)}
$$

where $N$ denotes the radial truncation order. In this work, $\left\|\mathbf{q}^{\prime}\right\|=30 \mathrm{~mm}^{-1}, x=E\left(\left\|\mathbf{q}^{\prime}\right\|\right) / E(0)=0.01$ and leads to the scale factor shown in Fig.5.

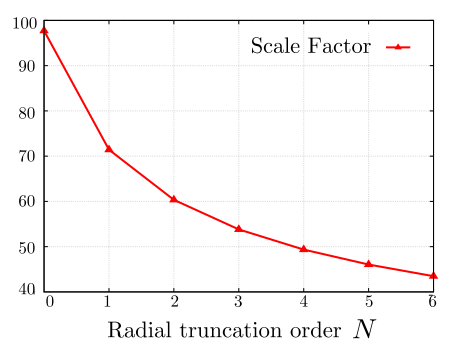

Figure 5: Scale factor $\zeta$ empiric evolution along truncation order $N$ with data attenuation $x=0.01$ and $\left\|\mathbf{q}^{\prime}\right\|=30 \mathrm{~mm}^{-1}$

\subsubsection{Ground Truth}

Fig. 6 shows the MR signal attenuation $E(\|\mathbf{q}\|)$ along a radial line $\left[0, q_{\max }\right]$. Two cases of data samples were studied: mono and bi-exponential decay. Diffusion coefficients were synthesized with $D_{i}=-\ln \left(x_{i}\right) / q_{\text {max }}^{2}$. For mono-exponential decay, we set $q_{\text {max }}=40, f_{1}=0, m_{1}=0, x_{1}=10^{-10}, D_{1} \approx 0.0144$. For bi-exponential decay, we set $q_{\max }=40, f_{1}=0.8, f_{2}=$ $0.2, m_{1}=0, m_{2}=20, x_{1}=10^{-10}, x_{2}=10^{-7}$ so that $D_{1} \approx$ $0.0144, D_{2} \approx 0.0101$. For the reconstruction of the MR signal, high resolution sampling protocol was used. The parameter $x$ is related to the scale factor $\zeta$ and can lead to erroneous values since it has to be set empirically. In this experiment, we arbitrary set the scale factor Eq.(24) with the signal attenuation to $x=0.01$. Consequently, this is a bad case scenario as the first term of the SPF reconstruction series $R_{0}$ has an ADC $D_{x}=0.0028$, i.e. 5 times less than the input data. However the reconstruction successfully converges to the true data in very few terms $n \approx 3$ (c.f. Fig. 6). This demonstrates the robustness and the stability of the estimation against wrong values of the scale factor. 


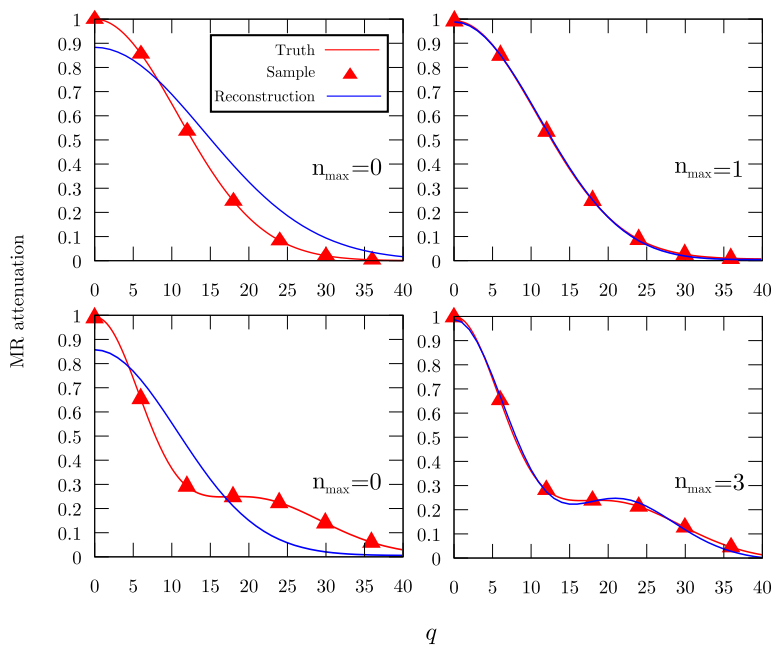

Figure 6: Radial reconstruction of signal $E$ using scaled Gaussian Laguerre functions. The scale $\zeta$ was arbitrary chosen to demonstrate the robustness of the reconstruction to wrong values. Top: Mono-exponential decay. Bottom: Bi-exponential decay.

Diffusion images from Fig.7 were synthesized following 3 sampling protocols: low ( 1 sphere, $\left.b=3000 \mathrm{~s} / \mathrm{mm}^{2}\right)$, medium (2 spheres, $\left.b=\{1000,3000\} \mathrm{s} / \mathrm{mm}^{2}\right)$ and high resolution (5 spheres, $b=\{500,1000,1700,2400,3000\} \mathrm{s} / \mathrm{mm}^{2}$ ) along with a single baseline image acquired at $b=0 \mathrm{~s} / \mathrm{mm}^{2}$. Each sphere is composed of 42 directions along the edges of a subdivided icosahedron. Estimation parameters were chosen empirically for each sampling protocol: low $\{N=0, L=4$, $\left.\zeta=100, \lambda_{N}=0, \lambda_{L}=10^{-6}\right\}$, medium $\{N=1, L=4$, $\left.\zeta=70, \lambda_{N}=\lambda_{L}=0\right\}$, high resolution $\{N=4, L=6, \zeta=50$, $\left.\lambda_{N}=\lambda_{L}=10^{-9}\right\}$. Fig. 7(b-c) demonstrates that our method can successfully reproduce the ODF obtained with the QBI approach, with a standard HARDI acquisition. Note that the resulting ODF is a sharp approximation of the true ODF. Besides, given the same dataset, we designed a PDF feature which simulate a HARDI acquisition with a higher gradient strength. This naturally leads to a sharper ODF (c.f. Fig.7d). In the same manner, Fig.7e illustrates the reconstruction of the true ODF using the corresponding PDF projection function. The results show a more precise representation of the estimated ODF. This is due to the Gaussian assumption of our model at low radial $N$ order which enables to better fit the synthetic data. We investigated the effect of increasing number of samples on the true ODF estimation at Fig.7(e-g). As expected the results exhibits successive accuracy improvements and converge to the true ODF shape Fig.7a. Using this synthetic experiments, we observed that more signal samples than our high resolution sampling protocol negligibly influence the estimation precision.

\subsubsection{Performance on noisy datasets}

In order to assess the robustness to noise of our proposed variational framework and simulate dMRI acquisitions, we added Rician noise of variance $\sigma$ to the signal $E$ which was then sampled into 29 values along the interval $q \in[0,30]$ as described above. Fig. 8 shows a comparison between the Gaus- sian and the Rician likelihood functions on a noisy dataset. On one hand, the Gaussian function is classically used in the least square minimization as in Eq.12 for its simplicity and its low computational cost, on the other hand it is not robust to noise since it creates undesirable radial oscillations at high $q$ values. On the contrary, the Rician likelihood function does not have this drawback and gives a correct estimation of $E$ (Fig.8e).

To investigate the benefit of the spatial regularization, we produced a numerical phantom of crossing fibers (horizontal and vertical networks) surrounded by water regions (upper left area) (c.f. Fig.9a). The Generalized Fractional Anisotropy (GFA) measure [20] was computed on the ODF. GFA is a generalization of the fractional anisotropy (FA) measure of DTI and is adequate to have hindsight on the global coherence of the dataset volume estimation. Indeed, every voxel is summarized by a scalar value (c.f. Fig.9b). In addition, each image was normalized independently to enhance the visualization contrast. This qualitative comparison highlights the need for spatial regularization within the estimation process. Indeed, when it comes to noisy data, anisotropic spatial regularization greatly improves the coherence of the volume estimation as illustrated in Fig.9c and 9d. It is worth noting that the gradient norm $\|\nabla \mathbf{A}\|$ is an adequate measure to set apart isotropic area from anisotropic area and subsequently, divergence $\operatorname{div}(\varphi\|\nabla \mathbf{A}\|)$ performs well in regularizing homogeneous area without degrading the discontinuities of the feature.

Fig.10 shows statistics on the performance of the PDE estimation with various likelihood and regularization functions $\psi$ and $\varphi$. It illustrates the PSNR (Peak Signal to Noise Ratio) of the reconstruction versus the quality of input datasets. The PSNR of the reconstruction stands for the PSNR between the known ground truth dataset volume $E$ and its estimation $\hat{E}$ in which values were restricted to $[0,1]$ in order to reflect the signal attenuation properties.

Out of the results of Fig.10a, the Rician likelihood function outperforms the Gaussian function and greatly improves the PSNR of the estimation. The explanation of this major improvements is to be found in the robustness of the Rician likelihood radial fit (c.f. Fig.8). Concerning the spatial regularization, Fig.10b shows the influence of regularization function $\varphi$ on the PSNR of the reconstruction $\hat{E}$. Although the results indicates modest improvements, the spatial regularization ensures numerical stability of the estimation by adding constraints when there are only very few samples available. This also brings stability to the fiber-tracking algorithms and helps to better estimate the white matter nerve fibers tracks [43].

\subsubsection{Influence of the q-space sampling scheme}

The number of data samples is limited because of the time required to acquire them. So the sampling scheme is critical and should be chosen wisely. Indeed, given a fixed number of samples (clinical constraint), which repartition of $q$-space samples is the best? Which radial order truncation $N$ should be chosen to fit Gaussian and bi-Gaussian MR datasets ? Which acquisition protocol gives the best results is the focus of the following experiments. 

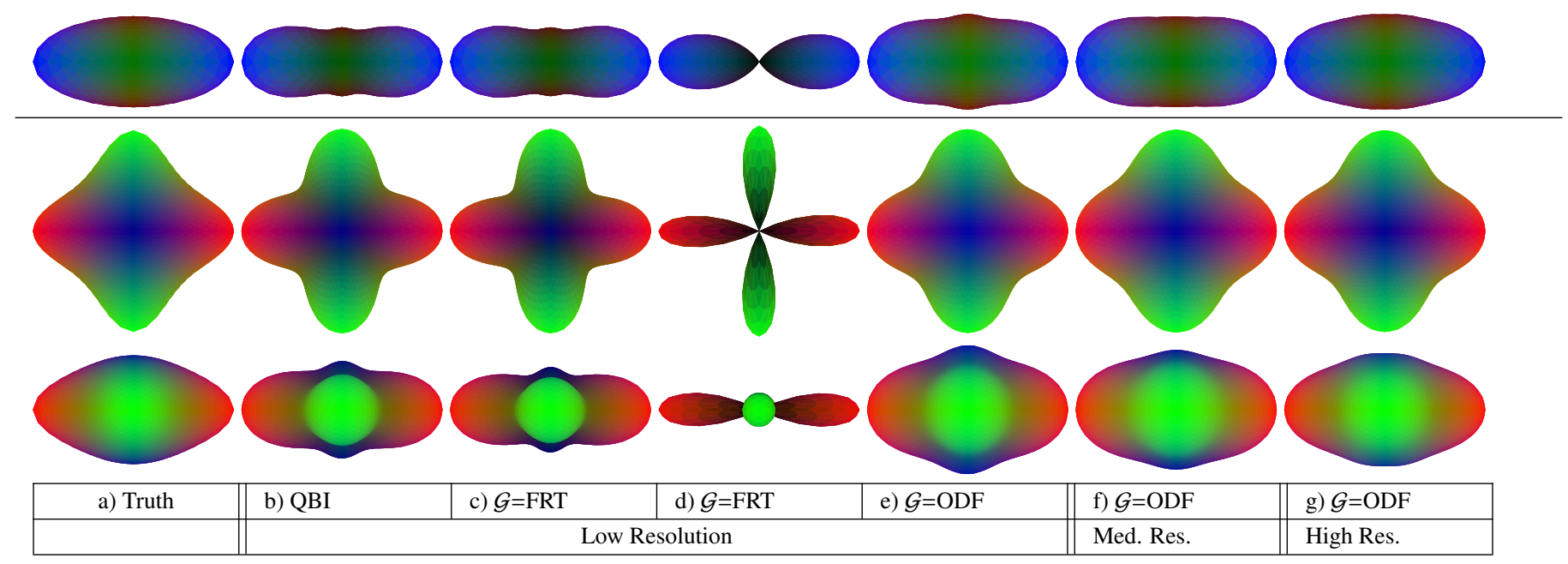

Figure 7: ODF comparisons: ODF versus QBI and ODF at low resolution versus ODF at high resolution. The first line corresponds to a single fiber direction $N_{b}=1$. The second and third lines correspond to crossing fibers $N_{b}=2$ respectively in face and profile view. From the left to the right: (a) True ODF computed analytically, (b) ODF given by QBI [40, 56], (c) simulation of QBI with our method, (d) FRT with higher $q^{\prime}$, (e-g) ODF estimations given by our method, with increasing number of samples.

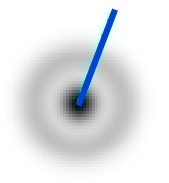

(a) Original Data

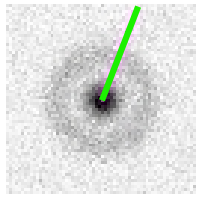

(b) Noisy data

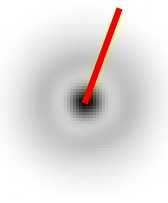

(c) Gaussian

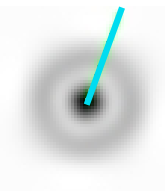

(d) Rician

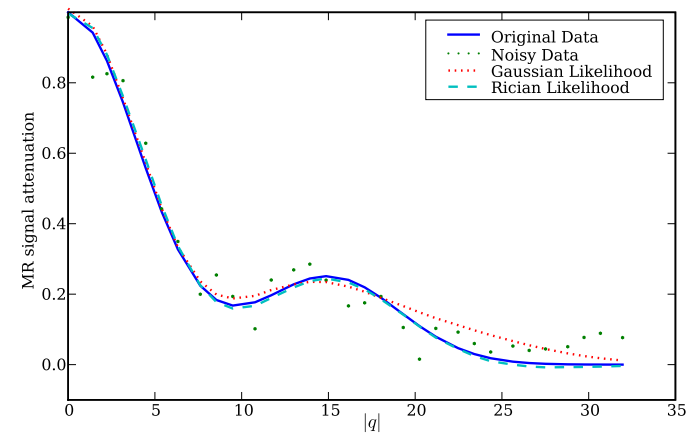

(e) Reconstruction of the radial profile

Figure 8: Reconstruction of isotropic diffusion signal $E$. Visualization of the radial attenuation profile along $\|\mathbf{q}\|$. Rician noise of $\sigma=0.1$ was added to the original data. Noisy data have a PNSR of $18.3 \mathrm{~dB}$.

Two sampling schemes were compared: non-uniform and uniform sampling of the $q$-space. Let $n_{s} \in \mathbb{N}$ be the total number of samples and $n_{b} \in \mathbb{N}$ the number of sampling sphere. Let $f$ be the number of samples on one sphere $x \in\left[1, n_{b}\right]$ so that

$$
f(\eta)=\frac{q_{x}^{\eta}}{\sum_{i=1}^{n_{b}} q_{i}^{\eta}} n_{s}
$$

where $q_{i}$ refers to the radius of the $i$-th sphere. The radius are distributed uniformly in the interval $\left[q_{\min }, q_{\max }\right]$. Then, $f(\eta=0)$ corresponds to the non-uniform sampling, with a constant number of samples on each sphere. The case $f(\eta=2)$ corresponds

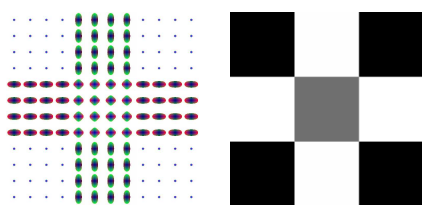

(a) Phantom: crossing fibers

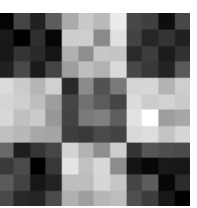

(c) Without

Regul.

PSNR: $12.8 \mathrm{~dB}$

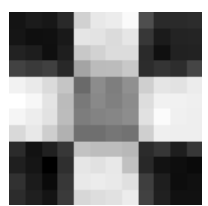

(d) With Regul. PSNR: $16.6 \mathrm{~dB}$
Figure 9: Effects of the spatial regularization on the Generalized Anisotropy (GFA) [20]. Isotropic area are black, anisotropic area are white. PSNR (Truth,Noisy) $=18.5 \mathrm{~dB}$. (a) The ODF of the synthetic phantom which is composed of two groups of fiber, horizontal and vertical, and surrounded by water. (b) GFA of the perfect dataset. (c) GFA of the LS estimation on a noisy dataset (without regularization). (d) GFA of the PDE estimation on the same noisy dataset (with regularization).

to the uniform sampling considering spherical normalization. Here $n_{s}=300, n_{b} \in[1,10]$, the scale factor $\zeta=80$ and the angular truncation order is set to $L=4$. The sampling points are as evenly spread on each sphere as possible and were computed by electrostatic energy minimization [72].

Fig.11(a) shows the evolution of $C$ the condition number Eq.(13) along with the number of sampling sphere $n_{b}$ and the radial truncation order $N$. Left and right figures respectively correspond to the non-uniform and uniform sampling. As expected, $C$ is very high when $N>n_{b}$ and leads to very unstable results. When $N \leq n_{b}$, the condition number increases slowly along with increasing values of $N$ and is quite constant along variations of $n_{b}$. Results using the non-uniform sampling exhibit more monotonous evolution than the uniform sampling. Fig.11(b) and (c) illustrate the PSNR evolution of the reconstruction of a Gaussian MR signal along variation of the same parameters. Although the maximum of the PSNR for both sampling protocols are quite the same $(\approx 40 \mathrm{~dB})$, it is clear that the non-uniform sampling protocol is more robust to wrong values 


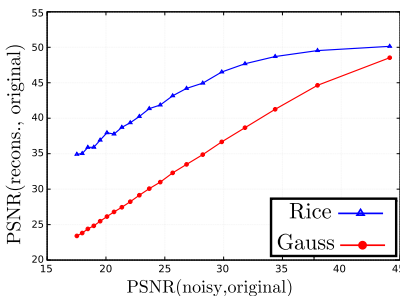

(a) Likelihood functions.

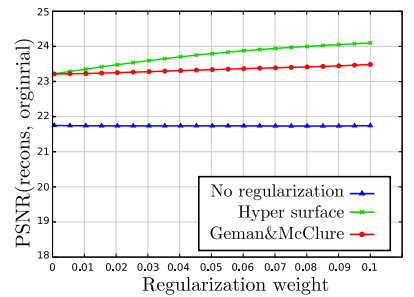

(b) Regularization functions.
Figure 10: Synthetic phantom of networks of crossing fibers (c.f. Fig.9a). (a) Evolution of the PSNR of the reconstruction $\hat{E}$ versus the PSNR of the input signal $E$ and different likelihood functions. (b) Evolution of the PSNR of $\hat{E}$ versus the regularization weight $\alpha_{r}$ and various regularization functions.

of $N$ and $n_{b}$. Besides, the robustness to wrong values of the scale factor $\zeta$ is represented by the lines of the figures. Therefore, as showed in Fig. 6, whereas the first order $N=0$ of the SPF basis has a Gaussian behavior, Gaussian data may not be well captured for very low $N$ because of wrong values of scale factor $\zeta$ not adapted to the signal decay. Once again, Fig.11(b) shows that the non-uniform sampling protocol is the most robust to wrong values of $\zeta$. Fig.11(d) shows the results on a bi-Gaussian noisy dataset, estimated using the damped linear least square Eq.(12). Besides the lower PSNR average compared to Fig.11(c), it is remarkable that best results of Fig.11(d) were also obtained from lower radial truncation order $N$ than Fig.11(c). Indeed, a reconstruction using high $N$ can significantly capture more noise than using lower $N$.

Out of the results, the non-uniform $(\eta=0)$ sampling protocol surprisingly gives better global results than the uniform proto$\operatorname{col}(\eta=2)$. The best reconstructions are obtained for $N \approx 3$ and $n_{b} \approx 4$. It confirms that the non-uniform sampling protocol is more efficient as it has a lot of samples on low $q$-space frequencies. It leads to a better reconstruction of the low frequencies of the MR signal and consequently a better reconstruction of the whole signal since low frequencies carry the greatest part of the MR signal energy.

Overall, there are two points to consider. First, as there is no certainty on the data model we want to retrieve, one should not distribute the samples according to a specific model. In other words, the samples should capture "unpredictible" variations of the MR signal. In Fig.11(b), the variations are mostly angular as the data represent a crossing fibers. Therefore the non-uniform sampling is more adequate as it defines many samples in the inner spheres. Second, the condition number given in Fig.11(a) indicates how ill-conditioned the matrix basis is, independently of the data. Out of this results, the non-uniform sampling seems to be more adequate to the SPF basis than the uniform sampling. Note that there are similar conclusions concerning the radii distributions in the $k$-space in the field of anatomical MRI [73].

\subsection{In-vivo experiments}

Our set of diffusion-weighted images were acquired in two spheres along 32 directions at $b=1000 \mathrm{~s} / \mathrm{mm}^{2}$ and $b=$ $3000 \mathrm{~s} / \mathrm{mm}^{2}$, and a single image at $b=0 \mathrm{~s} / \mathrm{mm}^{2}$. Thus, there were a total of 65 images acquired in a sequence of 15 minutes.

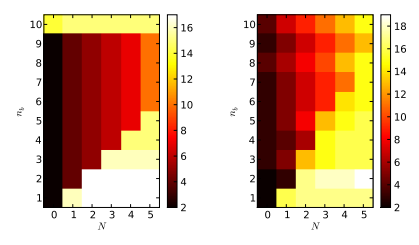

(a) Condition number. The lower the better.

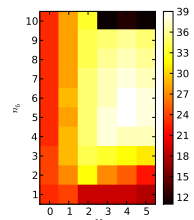

(c) Bi-Gaussian dataset. The higher the better.
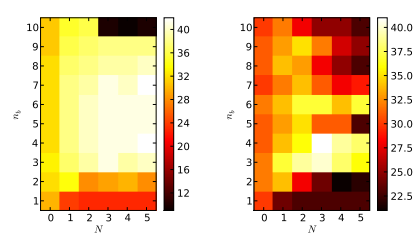

(b) Gaussian dataset.
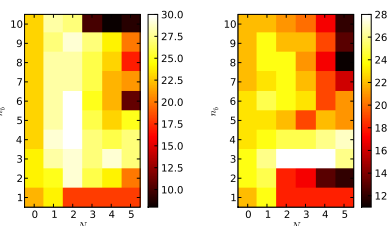

(d) Bi-Gaussian noisy dataset. The higher the better. The higher the better.

Figure 11: Comparison of constant vs adaptive sampling (respectively left and right image) on Gaussian and bi-Gaussian crossing fibers datasets. $N$ stands for the radial truncation in the SPF basis and $n_{b}$ is the number of sampling sphere in the $q$-space. (a) Influence on the $\log$ condition number $\log (C)$. (b) Reconstruction PSNR on a Gaussian dataset. (c) Reconstruction PSNR on a biGaussian dataset. (d) Reconstruction PSNR on a bi-Gaussian noisy dataset (PSNR (Truth,Noisy)=18.9 dB).

The SENSE parallel imaging protocol was used with a factor of acceleration set to 2 ; and only $80 \%$ of the $k$-space was acquired. Matrix size was $112 \times 112 \times 60$ and the image resolution was $2 \times 2 \times 2 \mathrm{~mm}^{3}$. Repetition time was $\mathrm{TR}=11490 \mathrm{~ms}$, echo time was $\mathrm{TE}=85 \mathrm{~ms}$. Time between two pulses and time of diffusion gradients were respectively $\Delta=42.2 \mathrm{~ms}$ and $\delta=26.3 \mathrm{~ms}$. The probability maps were computed by following the procedure described in Fig.2. Terms up to $N=1$ and $L=4$ were used in the calculations. Concerning computation time, the total time is less than a minute on a $3 \mathrm{Ghz}$ processor using the linear estimation Eq.(12). The variational framework is an iterative process which requires 70 minutes for approximately 200 iterations (c.f. Fig. 4). Although it is very penalizing in terms of computational time, is also computes far more precise estimators considering the Rician distribution of the noise, as shown in Fig. 10a. The computations includes calculations of SPF coefficients and projections along the 642 directions for the whole data-set $112 \times 112 \times 60$ volume.

The in-vivo data were processed using the Generalized Fractional Anisotropy (GFA) around the area of the corpus callosum (c.f. Fig.12). Additionally, the DTI and the ODF on the genu of the corpus callosum reveals the details of this anisotropy (c.f. Fig.13). As expected, DTI performs well in corpus callosum but fails in voxel with orientational heterogeneity as shown in Fig.13a. Therefore the FA at Fig.12b is reliable only inside corpus callosum. On the contrary, QBI can successfully retrieve multiple fibers orientations but is sensible to noise, especially in region of cerebrospinal fluid where the SNR of the MR signal is low (c.f. Fig.13b). Besides, the QBI is a sharp approximation of the true ODF and thus enhances the noise in the cerebrospinal region (c.f. Fig.12c). Whereas cerebrospinal fluid area are ex- 


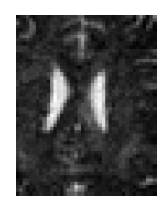

(a) $S_{0}$

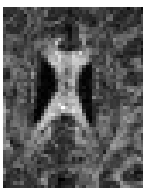

(e) Rice

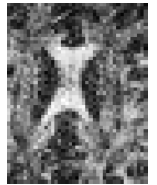

(b) DTI [5]

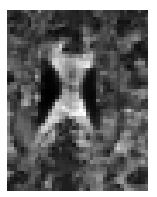

(f) Soft Reg.

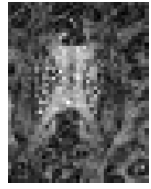

(c) QBI [56]

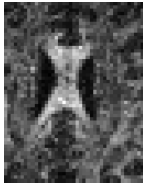

(d) $\mathcal{G}=\mathrm{ODF}$ [74]

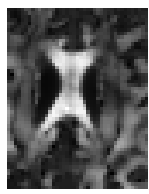

(g) Med Reg.

(h) Strong Reg.

$$
\begin{aligned}
& R_{0}(q)=\frac{2}{\pi^{1 / 4} \zeta^{3 / 4}} \exp \left(-\frac{q^{2}}{2 \zeta}\right) \\
& R_{1}(q)=\frac{32^{3 / 2}\left(1-\frac{2 q^{2}}{3 \zeta}\right)}{2 \sqrt{3} \pi^{1 / 4} \zeta^{3 / 4}} \exp \left(-\frac{q^{2}}{2 \zeta}\right) \\
& R_{2}(q)=\frac{152^{5 / 2}\left(-\frac{4 q^{2}}{3 \zeta}+\frac{4 q^{4}}{15 \zeta^{2}}+1\right)}{8 \sqrt{15} \pi^{1 / 4} \zeta^{3 / 4}} \exp \left(-\frac{q^{2}}{2 \zeta}\right) \\
& R_{3}(q)=\frac{35\left(-\frac{2 q^{2}}{\zeta}+\frac{4 q^{4}}{5 \zeta^{2}}-\frac{8 q^{6}}{105 \zeta^{3}}+1\right)}{2 \sqrt{35} \pi^{1 / 4} \zeta^{3 / 4}} \exp \left(-\frac{q^{2}}{2 \zeta}\right)
\end{aligned}
$$

Figure 12: Comparison of GFA [20] on region of corpus callosum and lateral ventricles. (a) Baseline image, $\mathbf{q}=0$. (b) DTI anisotropy map. (c) Q-Ball Imaging. (d) Previous work using damped least-square estimation. (e) Variational approach using Rician likelihood function. (f-g) Variational approach using Rician likelihood function + Hyper Surface regularization function.

pected to exhibits isotropic diffusion, the ODF obtained by the QBI method exhibits anisotropy. This may lead to wrong fiber reconstruction in fiber-tractography algorithms. On the contrary, the ODF obtained by our approach does not fall into this pitfall (Fig.12d), it successfully retrieves anisotropic shapes in brain white matter fibers regions and isotropic shape in cerebrospinal fluid area (c.f. Fig.13c).

\section{Discussion and conclusion}

The diffusion MRI challenge is to robustly estimate various features of the tissue micro-architecture using a highly reduced number of noisy samples. In this paper, we presented a method which tackles these issues by the use of a projection performed between a PDF feature and the diffusion signal in the Spherical Polar Fourier (SPF) basis. The direct consequence is that our approach can compute various features of the PDF without any further modification of the algorithm. Moreover, the SPF basis enables to reconstruct an accurate continuous MR signal with a reduced number of samples. Concerning the noise issue, we proposed a formulation of the estimation as an energy that simultaneously considers the Rician model of the MRI noise and regularization on spatial constraints. Our experiments indicate that our approach is able to reproduce results from the standard QBI method and also estimates more accurate ODF estimations. The results demonstrate the importance of the Rician likelihood term in the accuracy of the diffusion signal reconstruction. Since acquisition are usually averaged several times to increase the SNR, this could lead to the abandonment of this practice to the profit of more data samples.

\section{A. First radial $\boldsymbol{R}_{n}$}

We give the formula of the first radial functions $R_{n}, n \in[0,3]$ of the SPF basis.
We seek an eigenfunction $R_{n}$ with $n \in \mathbb{N}^{+}$so that low-order $R_{n}$ has a Gaussian behavior. We also want the basis made of the $R_{n}$ functions to be orthogonal and normalized according to spherical coordinates. From all the candidates, the generalized Laguerre polynomials $L_{n}^{l}$ form a orthogonal basis but do not fit the Gaussian and normalization constraints:

$$
\int_{q=0}^{\infty} \exp (-q) q^{l} L_{n}^{l}(q) L_{m}^{l}(q) d q=\delta_{n m} \frac{(l+n) !}{n !}
$$

Nonetheless, from this equation it is straightforward to see that $R_{n}$ made of normalized $L_{n}^{l}$ fit all the constraints. Let $n=m$, $l=1 / 2$ and $\rho=q^{2} / \zeta$, so that Eq.(30) is

$$
\int_{\rho=0}^{\infty} \exp (-\rho) \rho^{1 / 2} \frac{(n-1) !}{\Gamma(n+1 / 2)}\left[L_{n-1}^{1 / 2}(\rho)\right]^{2} d \rho=1
$$

where $d \rho=\frac{2 q}{\zeta} d q$ and the symbol $\zeta$ denotes the scale factor. Finally we have

$$
R_{n}(q, \zeta)=\left[\frac{2}{\zeta^{3 / 2}} \frac{n !}{\Gamma(n+3 / 2)}\right]^{1 / 2} \exp \left(-\frac{q^{2}}{2 \zeta}\right) L_{n}^{1 / 2}\left(\frac{q^{2}}{\zeta}\right),
$$

We can verify that we have spherical normalization

$$
\int_{q=0}^{\infty} R_{n}(q, \zeta) R_{m}(q, \zeta) q^{2} d q=\delta_{n m}
$$

\section{QBI}

Let $\mathcal{G}_{q^{\prime}}[E](\mathbf{k})$ be the Funk-Radon Transform (FRT) at vector $\mathbf{k}$ such as $\mathbf{k} \in \mathcal{S}^{2}$, where the $q$-space is restricted to the sphere of radius $q^{\prime}$. We denote $\mathbf{q}$ a vector of $q$-space such as $\mathbf{q}=\|\mathbf{q}\| \frac{\mathbf{q}}{\|\mathbf{q}\|}=$ $q \mathbf{u}$. For clarity reason, we denote $\sum_{n, l, m}^{\infty}=\sum_{n=0}^{\infty} \sum_{l=0}^{\infty} \sum_{m=-l}^{l}$. The FRT of $E$ on a sphere of radius $q^{\prime}$ and direction $\mathbf{k}$ is defined as:

$$
\begin{aligned}
\mathcal{G}_{q^{\prime}}[E](\mathbf{k}) & =\int_{\mathbf{u} \in \mathcal{S}^{2}} E\left(q^{\prime} \mathbf{u}\right) \delta\left(\mathbf{u}^{\mathrm{T}} \mathbf{k}\right) d \mathbf{u} \\
& =\int_{\mathbf{q} \in \mathbb{R}^{3}}\left[E(\mathbf{q}) \delta\left(q^{\prime}-q\right)\right]\left[\delta\left(\mathbf{u}^{\mathrm{T}} \mathbf{k}\right) \delta\left(q^{\prime}-q\right)\right] d \mathbf{q}
\end{aligned}
$$

\section{B. Radial eigen-functions: spherical normalization}




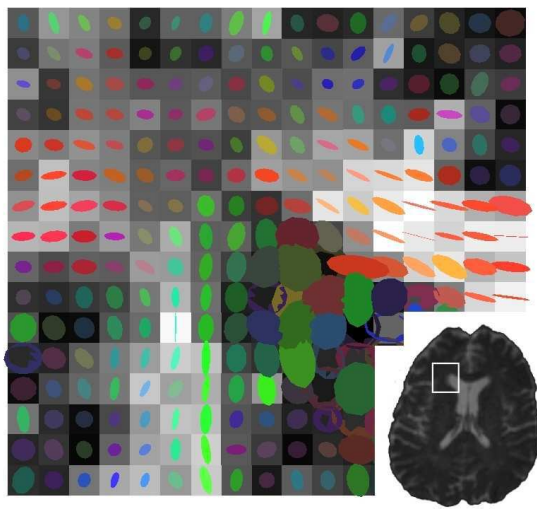

(a) DTI [5]

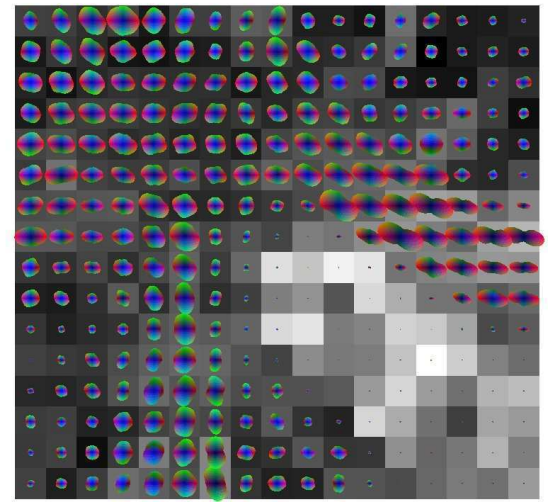

(b) QBI [56]

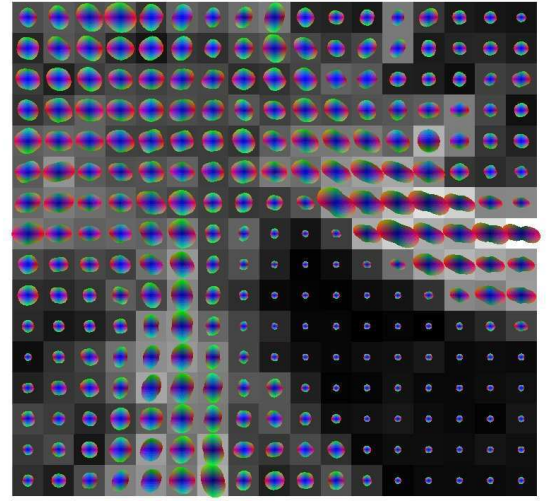

(c) Our method, $\mathcal{G}=\mathrm{ODF}$

Figure 13: In-vivo brain white matter ODF overlaid on GFA maps in a region of interest. $S_{0}$ image show the region of interest surrounding the corpus callosum-genu and the frontal horn, corpus callosum horizontal fibers appears from the right. DTI (a) and QBI (b) were computed with the outer sphere $b=3000 \mathrm{~s} / \mathrm{mm}^{2}$. Our method (c) shows the ODF obtained from both spheres $b=1000$ and $3000 \mathrm{~s} / \mathrm{mm}^{2}$.

We expand the previous expression into a series made of spherical harmonics $y_{l m}$ and radial functions $R_{n}(q)=q^{\prime-2} \delta\left(q^{\prime}-q\right)$,

$\mathcal{G}_{q^{\prime}}[E](\mathbf{k})=\int_{\mathbf{q} \in \mathbb{R}^{3}}\left[\sum_{n, l, m}^{\infty} b_{n, l, m} R_{n}(q) y_{l m}(\mathbf{u})\right]\left[\sum_{n^{\prime} l^{\prime} m^{\prime}}^{\infty} a_{n^{\prime} l^{\prime} m^{\prime}}^{\mathbf{k}} R_{n^{\prime}}(q) y_{l^{\prime} m^{\prime}}(\mathbf{u})\right] d \mathbf{q}$

Using Parseval's theorem, we express the FRT similarly to Eq.(15) as:

$$
\mathcal{G}_{q^{\prime}}[E](\mathbf{k})=\sum_{n, l, m}^{\infty} b_{n, l, m} a_{n, l, m}^{\mathbf{k}}
$$

where

$$
\begin{aligned}
b_{n, l, m} & =\int_{\mathbf{q} \in \mathbb{R}^{3}} E(\mathbf{q}) R_{n}(q) y_{l m}(\mathbf{u}) d \mathbf{q} \\
& =\int_{\mathbf{u} \in \mathcal{S}^{2}} E\left(q^{\prime} \mathbf{u}\right) y_{l m}(\mathbf{u}) d \mathbf{u} \\
a_{n, l, m}^{\mathbf{k}} & =\int_{\mathbf{q} \in \mathbb{R}^{3}} \delta\left(\mathbf{u}^{\mathrm{T}} \cdot \mathbf{k}\right) \delta\left(q^{\prime}-q\right) R_{n}(q) y_{l m}(\mathbf{u}) d \mathbf{q} \\
& =2 \pi P_{l}(0) y_{l m}(\mathbf{k}) \quad(\text { Funk-Hecke theorem }[40,56])
\end{aligned}
$$

Note that Tuch demonstrated in [21] that the FRT of the signal $E$ is expressed in the displacement space as:

$$
\mathcal{G}_{q^{\prime}}[E](\mathbf{k})=\int_{\mathbf{p} \in \mathbb{R}^{3}} P(\mathbf{p}) 2 \pi q^{\prime} J_{0}\left(2 \pi q^{\prime}\|\mathbf{p}\|\right) \delta\left(1-\frac{\mathbf{p}^{\mathrm{T}} \mathbf{k}}{\|\mathbf{p}\|}\right) d \mathbf{p}
$$

Similarly to our framework, we see from Eq.(36) that QBI can be expressed with a basis made of real SH $y_{l m}$ and radial functions $R_{n}(q)=q^{\prime-2} \delta\left(q^{\prime}-q\right)$. In this basis, $H_{\mathbf{k}}(\mathbf{p})=$ $2 \pi q^{\prime} J_{0}\left(2 \pi q^{\prime} p\right)$ and $a_{n, l, m}^{\mathbf{k}}=2 \pi P_{l}(0) y_{l m}(\mathbf{k})$.

\section{DOT}

The Diffusion Orientation Tensor (DOT) [26] method aims to compute the PDF $P\left(R_{0} \mathbf{k}\right)$ at radius $R_{0}$, for all orientation of the unit sphere $\mathbf{k} \in \mathcal{S}^{2}$. We can express the DOT in the displacement probability space as:

$$
P\left(R_{0} \mathbf{k}\right)=\int_{\mathbf{p} \in \mathbb{R}^{3}} P(\mathbf{p}) \delta(\mathbf{k}-\mathbf{p}) d \mathbf{p}
$$

Using Parseval's theorem, we express the DOT in the $q$-space as:

$$
P\left(R_{0} \mathbf{k}\right)=\int_{\mathbf{q} \in \mathbb{R}^{3}} E(\mathbf{q}) \exp \left(i 2 \pi R_{0} q \mathbf{k}^{\mathrm{T}} \mathbf{u}\right) d \mathbf{q}
$$

Using the plane wave expansion, we have:

$$
\begin{aligned}
P\left(R_{0} \mathbf{k}\right) & =\int_{\mathbf{q} \in \mathbb{R}^{3}}[E(\mathbf{q})]\left[\sum_{l^{\prime} m^{\prime}} 4 \pi i^{l^{\prime}} j_{l^{\prime}}\left(2 \pi q R_{0}\right) y_{l^{\prime} m^{\prime}}(\mathbf{k}) y_{l^{\prime} m^{\prime}}(\mathbf{u})\right] d \mathbf{q} \\
& =\int_{\mathbf{q} \in \mathbb{R}^{3}}\left[\sum_{n, l, m}^{\infty} b_{n, l, m} R_{n}(q) y_{l m}(\mathbf{u})\right]\left[\sum_{n^{\prime} l^{\prime} m^{\prime}}^{\infty} a_{n^{\prime} l^{\prime} m^{\prime}}^{\mathbf{k}} R_{n^{\prime}}(q) y_{l^{\prime} m^{\prime}}(\mathbf{u})\right] d \mathbf{q}
\end{aligned}
$$

where $R_{n}(q)=j_{l}\left(2 \pi q R_{0}\right) \delta_{n l}$. Using Parseval's theorem once more, we express the DOT similarly to Eq.(15) as:

$$
P\left(R_{0} \mathbf{k}\right)=\sum_{n, l, m}^{\infty} b_{n, l, m} a_{n, l, m}^{\mathbf{k}}
$$

where

$$
\begin{aligned}
b_{n, l, m} & =\int_{\mathbf{q} \in \mathbb{R}^{3}} E(\mathbf{q}) R_{n}(q) y_{l m}(\mathbf{u}) d \mathbf{q}=\int_{\mathbf{u} \in \mathcal{S}^{2}} \frac{I_{l}(\mathbf{u})}{4 \pi} y_{l m}(\mathbf{u}) d \mathbf{u} \\
I_{l}(\mathbf{u}) & =\frac{R_{0}^{l} \Gamma\left(\frac{l+3}{2}\right)}{2^{l+3} \pi^{3 / 2}\left(-\ln (E(\mathbf{u})) 4^{-1}(\pi q)^{-2}\right)^{(l+3) / 2} \Gamma(l+3 / 2)} \\
& \times 1 F 1\left(\frac{l+3}{2} ; l+\frac{3}{2} ; \frac{R_{0}^{2}}{\ln (E(\mathbf{u}))(\pi q)^{-2}}\right)
\end{aligned}
$$

(Spherical Bessel transform Eq.27[26])

$a_{n, l, m}^{\mathbf{k}}=4 \pi i^{l} y_{l m}(\mathbf{k})$ 
Similarly to our framework, we see from Eq.(44) that DOT can be expressed with a basis made of real SH $y_{l m}$ and radial functions $R_{n}(q)=j_{l}\left(2 \pi q R_{0}\right) \delta_{n l}$. In this basis, $H_{\mathbf{k}}(\mathbf{p})=\delta(\mathbf{k}-\mathbf{p})$ and $a_{n, l, m}^{\mathbf{k}}=4 \pi i^{l} y_{l m}(\mathbf{k})$.

\section{E. Relation of SPF to Hermite polynomials}

Ozarslan et al. proposed in [55] to reconstruct the radial part of the diffusion signal using a series made of Hermite polynomials. In this section, we express the relationship between our SPF basis and Ozarslan et al.'s approach. Hermite polynomials are eigen functions of the Fourier transform. Recall that the Hermite polynomials $H_{n}$ are related to the Laguerre polynomials by the following equation:

$$
H_{2 n+1}(x)=(-1)^{n} 2^{2 n+1} n ! x L_{n}^{1 / 2}\left(x^{2}\right)
$$

Let $q=x \sqrt{\zeta}$, we have:

$$
L_{n}^{1 / 2}\left(\frac{q^{2}}{\zeta}\right)=\frac{(-1)^{n}}{2^{2 n+1} n !} \frac{\zeta^{1 / 2}}{q} H_{2 n+1}\left(\frac{q}{\zeta^{1 / 2}}\right)
$$

Finally we have

$R_{n}(q)=\left[\frac{n !}{\Gamma(n+3 / 2)}\right]^{1 / 2} \frac{2^{-2 n-1 / 2}(-1)^{n}}{\zeta^{1 / 4} n !} \frac{1}{q} \exp \left(\frac{-q^{2}}{2 \zeta}\right) H_{2 n+1}\left(\frac{q}{\zeta^{1 / 2}}\right)$

\section{F. Real Wigner matrix rotation}

In this section, we express the mathematical tools necessary to implement the rotation of real spherical harmonics. Under a rotation transformation expressed in terms of the Euler angles $(\alpha, \beta, \gamma)$, the rotated complex spherical harmonics $Y_{l}^{m}$ can be expressed as a linear combination of $Y_{l}^{m^{\prime}}$.

$$
\operatorname{Rot}_{\alpha, \beta, \gamma}\left[Y_{l}^{m}(\theta, \phi)\right]=\sum_{m^{\prime}=-l}^{l} Y_{l}^{m^{\prime}}(\theta, \phi)\left[e^{-i m^{\prime} \alpha} d_{m^{\prime} m}^{(l)}(\beta) e^{-i m \gamma}\right]
$$

where the $d$-small Wigner matrix [75] is:

$$
\begin{aligned}
& d_{m^{\prime} m}^{(l)}(\beta)=\left[\frac{\left(l+m^{\prime}\right) !\left(l-m^{\prime}\right) !}{(l+m) !(l-m) !}\right]^{1 / 2} \\
& \sum_{k=\max \left(0, m-m^{\prime}\right)}^{\min \left(l-m^{\prime}, l+m\right)}\left[(-1)^{k+m^{\prime}-m}\left(\begin{array}{c}
l+m \\
k
\end{array}\right)\left(\begin{array}{c}
l-m \\
l-m^{\prime}-k
\end{array}\right)\right. \\
& \left.(\cos \beta / 2)^{2 l+m-m^{\prime}-2 k}(\sin \beta / 2)^{2 k+m^{\prime}-m}\right]
\end{aligned}
$$

We are interested in the real Wigner rotation matrix $D_{m^{\prime} m}^{(l)}$ so that

$$
\operatorname{Rot}_{\alpha, \beta, \gamma}\left[y_{l}^{m}(\theta, \phi)\right]=\sum_{m^{\prime}=-l}^{l} y_{l}^{m^{\prime}}(\theta, \phi) D_{m^{\prime} m}^{(l)}(\alpha, \beta, \gamma)
$$

Recall that the real SH can be expressed as a linear combination of the complex $\mathrm{SH}$, this leads to [76], appendix B.4:

$$
D_{m^{\prime} m}^{(l)}= \begin{cases}d_{m^{\prime} m}^{(l)}(\beta) \cos (F)+(-1)^{m^{\prime}} d_{-m^{\prime} m}^{(l)}(\beta) \cos (G) & m^{\prime} \geq 0, m \geq 0 \\ (-1)^{m^{\prime}+1} d_{m^{\prime} m}^{(l)}(\beta) \sin (F)+d_{-m^{\prime} m}^{(l)}(\beta) \sin (G) & m^{\prime}<0, m \geq 0 \\ (-1)^{m} d_{m^{\prime} m}^{(\beta)} \sin (F)+(-1)^{m+m^{\prime}} d_{-m^{\prime} m}^{(l)}(\beta) \sin (G) & m^{\prime} \geq 0, m<0 \\ (-1)^{m+m^{\prime}} d_{m^{\prime} m}^{(l)}(\beta) \cos (F)+(-1)^{m+1} d_{-m^{\prime} m}^{(l)}(\beta) \cos (G) & m^{\prime}<0, m<0\end{cases}
$$

where $F=m \gamma+m^{\prime} \alpha$ and $G=m \gamma-m^{\prime} \alpha$.

\section{G. Gradient}

We demonstrate here that $\|\nabla \mathbf{E}\|=\|\nabla \mathbf{A}\|$ where $\mathbf{E}$ is the diffusion MR signal and $\mathbf{A}$ is its coefficients in the SPF basis so that $\Psi_{n, l, m}(\mathbf{q})=R_{n}(\|\mathbf{q}\|) y_{l}^{m}\left(\frac{\mathbf{q}}{\|\mathbf{q}\|}\right)$. Let $\mathbf{x}$ be a voxel of the data volume, i.e. $\mathbf{x} \in \Omega$.

$$
\begin{aligned}
\|\nabla \mathbf{E}\| & =\int_{\mathbf{q} \in \mathbb{R}^{3}}\left[\frac{\partial \mathbf{E}(\mathbf{q})}{\partial \mathbf{x}}\right]^{2} d \mathbf{q} \\
& =\int_{\mathbf{q} \in \mathbb{R}^{3}}\left[\sum_{n, l, m} \Psi_{n, l, m}(\mathbf{q}) \frac{\partial a_{n, l, m}}{\partial \mathbf{x}}\right]^{2} d \mathbf{q} \\
& =\int_{\mathbf{q} \in \mathbb{R}^{3}} \sum_{n, l, m}\left[\Psi_{n, l, m}(\mathbf{q}) \frac{\partial a_{n, l, m}}{\partial \mathbf{x}}\right]^{2} d \mathbf{q} \\
& +2 \int_{\mathbf{q} \in \mathbb{R}^{3}} \sum_{n, l, m}\left[\Psi_{n, l, m}(\mathbf{q}) \frac{\partial a_{n, l, m}}{\partial \mathbf{x}}\left[\sum_{n^{\prime}, l^{\prime}, m^{\prime}}^{(n, l, m)-1} \Psi_{n^{\prime}, l^{\prime}, m^{\prime}}(\mathbf{q}) \frac{\partial a_{n^{\prime}, l^{\prime}, m^{\prime}}}{\partial \mathbf{x}}\right]\right] d \mathbf{q}
\end{aligned}
$$

Since $\Psi$ is an orthonormal basis and all voxel $\mathbf{x}$ have the same basis, this leads to:

$$
\begin{aligned}
\|\nabla \mathbf{E}\| & =\int_{\mathbf{q} \in \mathbb{R}^{3}} \sum_{n, l, m}\left[\frac{\partial a_{n, l, m}}{\partial \mathbf{x}}\right]^{2}\left[\Psi_{n, l, m}(\mathbf{q})\right]^{2} d \mathbf{q} \\
& =\sum_{n, l, m}\left[\frac{\partial a_{n, l, m}}{\partial \mathbf{x}}\right]^{2} \\
& =\|\nabla \mathbf{A}\|
\end{aligned}
$$

\section{Acknowledgments}

The authors would like to thank Frank Lamberton, Bernard Mazoyer from GIN/Cyceron for their dataset and the fruitful discussion.

\section{References}

[1] D. LeBihan, E. Breton, D. Lallemand, P. Grenier, E. Cabanis, M. LavalJeantet, Mr imaging of intravoxel incoherent motions: Application to diffusion and perfusion in neurologic disorders, Radiology (1986) 401-407.

[2] D. Le Bihan, E. Breton, D. Lallemand, Perfusion in intravoxel incoherent motion mr imaging, Radiology 168 (1988) 497-505.

[3] M. Moseley, Y. Cohen, J. Mintorovitch, L. Chileuitt, H. Shimizu, J. Kucharczyk, M. Wendland, P. Weinstein, Early detection of regional cerebral ischemia in cats: comparison of diffusion and t 2-weighted mri and spectroscopy, Magn. Res. Med. 14 (2) (1990) 330-346.

[4] E. Stejskal, J. Tanner, Spin diffusion measurements: spin echoes in the presence of a time-dependent field gradient, Journal of Chemical Physics 42 (1965) 288-292.

[5] P. J. Basser, J. Mattiello, D. LeBihan, Estimation of the effective selfdiffusion tensor from the nmr spin echo, Journal of Magnetic Resonance 103 (1994) 247-254.

[6] B. Jian, B. C. Vemuri, E. Özarslan, P. R. Carney, T. H. Mareci, A novel tensor distribution model for the diffusion-weighted mr signal, NeuroImage 37 (2007) 164-176.

[7] L. Frank, Characterization of anisotropy in high angular resolution diffusion-weighted mri, Magn. Res. Med. 47 (2002) 1083-1099.

[8] P. Callaghan, Principles of Nuclear Magnetic Resonance Microscopy, Oxford University Press, USA, 1991.

[9] D. Cory, A. Garroway, Measurement of translational displacement probabilities by nmr: an indicator of compartmentation, Magn. Res. Med. 14 (3) (1990) 435-444. 
[10] P. Basser, Relationships between diffusion tensor and q-space mri, Magn. Res. Med. 47 (2) (2002) 392-397.

[11] V. Wedeen, T. Reese, D. Tuch, M. Weigl, J.-G. Dou, R. Weiskoff, D. Chesler, Mapping fiber orientation spectra in cerebral white matter with fourier transform diffusion mri, ISMRM, 2000, p. 82.

[12] J. Tanner, E. Stejskal, Restricted self-diffusion of protons in colloidal systems by the pulsed-gradient, spin-echo method, Journal of Chemical Physics 49 (1968) 1768-1777.

[13] J. Tanner, Transient diffusion in a system partitioned by permeable barriers. application to nmr measurements with a pulsed field gradient, Journal of Chemical Physics 69 (1978) 1748.

14] P. Kuchel, A. Coy, P. Stilbs, Nmr diffusion-diffraction of water revealing alignment of erythrocytes in a magnetic field and their dimensions and membrane transport characteristics, Magn. Res. Med. 37 (5) (1997) 637 643.

[15] M. King, J. Houseman, D. Gadian, A. Connelly, Localized q-space imaging of the mouse brain, Magn. Res. Med. 38 (6) (1997) 930-937.

[16] P. Callaghan, A. Coy, D. MacGowan, K. Packer, F. Zelaya, Diffractionlike effects in nmr diffusion studies of fluids in porous solids, Nature 351 (6326) (1991) 467-469.

[17] P. W. Kuchel, C. J. Durrant, Permeability coefficients from nmr q-space data: Models with unevenly spaced semi-permeable parallel membranes, Journal of Magnetic Resonance 139 (1999) 258-272.

[18] D. Alexander, Axon radius measurements in vivo from diffusion mri: a feasible study, MMBIA, 2007, pp. 1-8.

[19] E. Özarslan, P. J. Basser, Mr diffusion-'diffraction' phenomenon in multipulse-field-gradient experiments, Journal of Magnetic Resonance 188 (2) (2007) 285-294.

[20] D. Tuch, R. Weisskoff, J. Belliveau, V. Wedeen, High angular resolution diffusion imaging of the human brain, ISMRM, 1999, p. 321

[21] D. Tuch, Q-ball imaging, Magn. Res. Med. 52 (2004) 1358-1372.

[22] P. Basser, C. Pierpaoli, A Simplified Method to Measure the Diffusion Tensor from Seven MR Images, Magn. Res. Med. 39 (1998) 928-934.

[23] P. Basser, S. Pajevic, C. Pierpaoli, J. Duda, A. Aldroubi, In vivo fiber tractography using dt-mri data, Magn. Res. Med. 44 (4) (2000) 625-632.

[24] D. A. Yablonskiy, G. L. Bretthorst, J. J. Ackerman, Statistical model for diffusion attenuated mr signal, Magn. Res. Med. 50 (2003) 664-669.

[25] J. Tournier, F. Calamante, D. Gadian, A. Connely, Direct estimation of the fiber orientation density function from diffusion-weighted mri data using spherical deconvolution, NeuroImage 23 (2004) 1179-1185.

[26] E. Özarslan, T. M. Sherperd, B. C. Vemuri, S. J. Blackband, T. H. Mareci, Resolution of complex tissue microarchitecture using the diffusion orientation transform (dot), NeuroImage 31 (2006) 1086-1103.

[27] Y.-C. Wu, A. L. Alexander, Hybrid diffusion imaging, NeuroImage 36 (3) (2007) $617-629$.

[28] C. Liu, R. Bammer, B. Acar, M. Moseley, Characterizing non-gaussian diffusion by using generalized diffusion tensors, Magn. Res. Med. 51 (2004) 924-937.

[29] E. Ozarslan, T. Mareci, Generalized diffusion tensor imaging and analytical relationships between diffusion tensor imaging and high angular resolution diffusion imaging, Magn. Res. Med. 50 (5) (2003) 955-965.

[30] Y. Assaf, P. J. Basser, Composite hindered and restricted model of diffusion (charmed) mr imaging of the human brain, NeuroImage 27 (2005) $48-58$.

[31] M. Khachaturian, J. Wisco, D. Tuch, Boosting the sampling efficiency of q-ball imaging using multiple wavevector fusion, Magn. Res. Med. 57 (2007) 289-296.

[32] V. Pickalov, P. J. Basser, 3d tomographic reconstruction of the average propagator from mri data, ISBI, 2006, pp. 710-713.

[33] P. Morris, Nuclear Magnetic Resonance Imaging in Medicine and Biology, Oxford University Press, USA, 1986.

[34] J. Libove, J. Singer, Resolution and signal-to-noise relationships in nmr imaging in the human body, Journal of Physics E: Scientific Instruments 13 (1980) 38-44.

[35] L. Breiman, Probability addison-wesley, Reading, Mass.

[36] H. Gudbjartsson, S. Patz, The rician distribution of noisy mri data, Magn. Res. Med. 34 (1995) 910-914.

[37] R. Henkelman, Measurement of signal intensities in the presence of noise in mr images, Med. Phys. 12 (1985) 232-233.

[38] J. Sijbers, A. den Dekker, J. van Audekerke, M. Verhoye, D. van Dyck, Estimation of the noise in magnitude $\mathrm{mr}$ images, Magnetic Resonance
Imaging 16 (1998) 87-90.

[39] D. Jones, P. Basser, "squashing peanuts and smashing pumpkins": How noise distorts diffusion-weighted mr data, Magn. Res. Med. 52 (5) (2004) 979-993.

[40] C. Hess, P. Mukherjee, E. Han, D. Xu, D. Vigneron, Q-ball reconstruction of multimodal fiber orientations using the spherical harmonic basis., Magn. Res. Med. 56 (1) (2006) 104-117.

[41] M. Descoteaux, N. Wiest-Daessle, S. Prima, C. Barillot, R. Deriche, Impact of rician adapted non-local means filtering on hardi, in: MICCAI, New York/USA, 2008, pp. 122-130.

[42] N. Wiest-Daessle, S. Prima, P. Coupe, S. Morrissey, C. Barillot, Rician noise removal by non-local means filtering for low signal-to-noise ratio mri: Application to dt-mri, in: MICCAI, New York/USA, 2008, pp. 104 117.

[43] H. Assemlal, D. Tschumperlé, L. Brun, Fiber tracking on hardi data using robust odf fields, ICIP, 2007, pp. 133-136.

[44] S. Huzinaga, Gaussian-type functions for polyatomic systems. i, Journal of Chemical Physics 42 (1965) 1293-1302.

[45] T. Zivkovic, Z. Maksic, Explicit Formulas for Molecular Integrals over Hermite-Gaussian Functions, Journal of Chemical Physics 49 (1968) 3083-3087.

[46] D. W. Ritchie, High-order analytic translation matrix elements for realspace six-dimensional polar fourier correlations, J. Appl. Cryst. 38 (2005) 808-818.

[47] G. Andrews, R. Askey, R. Roy, Special Functions, Cambridge University Press, 1999.

[48] D. Alexander, G. Barker, S. Arridge, Detection and modeling of nongaussian apparent diffusion coefficient profiles in human brain data, Magnetic Resonance Imaging 48 (2002) 331-340.

[49] C. Clark, D. Le Bihan, Water diffusion and anisotropy at high b values in the human brain, Magn. Res. Med. 44 (2000) 852-859.

[50] Y. Cohen, Y. Assaf, High b-value q-space analyzed diffusion-weighted mrs and mri in neuronal tissues - a technical review, NMR Biomed 15 (2002) 516-542.

[51] T. Niendorf, R. Dijkhuizen, N. D.G., C. M. van Lookeren, K. Nicolay, Biexponential diffusion attenuation in various states of brain tissue: implications for diffusion-weighted imaging, Magn. Res. Med. 36 (1996) 847-857.

[52] Y. Assaf, Y. Cohen, In vivo and in vitro bi-exponential diffusion of nacetyl aspartate (naa) in rat brain: a potential structural probe?, NMR Biomed 11 (1998) 67-74.

[53] R. Mulkern, H. Zengingonul, R. Robertson, P. Bogner, K. Zou, H. Gudbjartsson, C. Guttmann, D. Holtzman, W. Kyriakos, F. Jolesz, et al., Multicomponent apparent diffusion coefficients in human brain: Relationship to spin-lattice relaxation, Magn. Res. Med. 44 (2) (2000) 292-300.

[54] M. Abramowitz, I. Stegun, Handbook of mathematical functions with formulas, graphs, and mathematical tables. national bureau of standards applied mathematics series 55. tenth printing.

[55] E. Ozarslan, C. Koay, P. Basser, Simple harmonic oscillator based estimation and reconstruction for one-dimensional q-space, Vol. 16, MR. In Proc. Intl. Soc. Mag. Reson. Med., 2008, p. 35.

[56] M. Descoteaux, E. Angelino, S. Fitzgibbons, R. Deriche, Regularized, fast and robust analytical q-ball imaging, Magn. Res. Med. 58 (2007) 497-510.

[57] M. Descoteaux, E. Angelino, S. Fitzgibbons, R. Deriche, Apparent diffusion coefficients from high angular resolution diffusion imaging: estimation and applications., Magn Reson Med 56 (2) (2006) 395-410.

[58] D. Healy, H. Hendriks, P. Kim, Spherical deconvolution, Journal of Multivariate Analysis 67 (1) (1998) 1-22.

[59] M. A. Blanco, M. Flórez, M. Bermejo, Evaluation of the rotation matrices in the basis of real spherical harmonics, J. Mol. Struct. 419 (1997) 19-27.

[60] M. Bernstein, D. Thomasson, W. Perman, Improved detectability in low signal-to-noise ratio magnetic resonance images by means of a phasecorrected real reconstruction, Medical Physics 16 (1989) 813.

[61] W. Edelstein, P. Bottomley, L. Pfeifer, A signal-to-noise calibration procedure for NMR imaging systems, Medical Physics 11 (1984) 180.

[62] D. Tschumperlé, R. Deriche, Tensor field visualization with pde's and application to dt-mri fiber visualization, VLSM, Nice, France, 2003.

[63] C. Chefd'hotel, D. Tschumperlé, R. Deriche, O. Faugeras, Regularizing flows for constrained matrix-valued images, J. Math. Imaging Vis. 20 (12) (2004) 147-162. 
[64] O. Pasternak, N. Sochen, Y. Assaf, Visualization and Image Processing of Tensor Fields, J.Weickert, H.Hagen, 2005.

[65] A. Ramirez-Manzanares, M. Rivera, A method for estimating brain nerve bundles, by restoring and filtering intra-voxel information in diffusion tensor mri data, VLSM, Nice, France, 2003.

[66] Y. Chen, W. Guo, Q. Zeng, X. Yan, F. Huang, H. Zhang, G. He, B. Vemuri, Y. Liu, Estimation, smoothing, and characterization of apparent diffusion coefficient profiles from high angular resolution dwi, CVPR 1 (2004) 588-593.

[67] Z. Wang, B. Vemuri, Y. Chen, T. Mareci, A constrained variational principle for direct estimation and smoothing of the diffusion tensor field from complex dwi, Transactions on Medical Imaging (2004) 930-939.

[68] S. Basu, T. Fletcher, R. Whitaker, Rician noise removal in diffusion tensor mri, MICCAI (9(Pt 1)) (2006) 117-125.

[69] B. Murphy, P. Carson, J. Ellis, Y. Zhang, R. Hyde, T. Chenevert, Signalto-noise measures for magnetic resonance imagers, Magnetic Resonance Imaging 11 (3) (1993) 425-428.

[70] L. Kaufman, D. Kramer, Measuring signal-to-noise ratios in mr imaging, Radiology 173 (1989) 265-267.

[71] J. Sijbers, D. Poot, A. Dekker, W. Pintjens, Automatic estimation of the noise variance from the histogram of a magnetic resonance image, Physics in Medecine and Biology 52 (5) (2007) 1335.

[72] A. S. D.K. Jones, M.A. Horsfield, Optimal strategies for measuring diffusion in anisotropic systems by magnetic resonance imaging, Magn. Res. Med. 42 (3) (1999) 515-525.

[73] M. Lustig, D. Donoho, J. M. Pauly, Sparse mri: The application of compressed sensing for rapid mr imaging, Magn. Res. Med. 58 (6) (2007) 1182-1195.

[74] H. Assemlal, D. Tschumperlé, L. Brun, Efficient computation of pdfbased characteristics from diffusion $\mathrm{mr}$ signal., in: MICCAI, New York/USA, 2008, pp. 70-78.

[75] Z. Su, P. Coppens, Rotation of real spherical harmonics, Foundations of Crystallography 50 (5) (1994) 7673.

[76] D. Ritchie, Parametric protein shape recognition, Ph.D. thesis, University of Aberdeen, U.K. (1998). 